This is an Author's Accepted Manuscript of an article published as: COSTA, J.L. (2010) On the classification of stationary electro-vacuum black holes. Classical and Quantum Gravity. 27(3):0350103 available online at:

http://dx.doi.org/10.1088/0264-9381/27/3/035010 


\title{
ON THE CLASSIFICATION OF STATIONARY ELECTRO-VACUUM BLACK HOLES
}

\author{
by
}

João Lopes Costa

\begin{abstract}
We obtain a classification of stationary, $I^{+}$-regular, non-degenerate and analytic electro-vacuum space-times in terms of Weinstein solutions. In particular, for connected horizons, we prove uniqueness of the Kerr-Newman black holes.
\end{abstract}

\section{Contents}

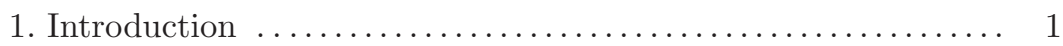

2. Preliminaries ......................................

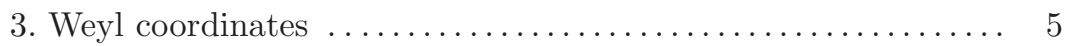

4. Reduction to a harmonic map problem ............... 9

4.1. Distance function on the target manifold ........... 10

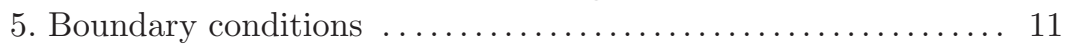

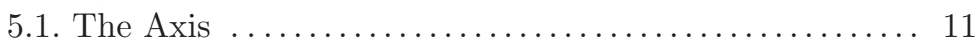

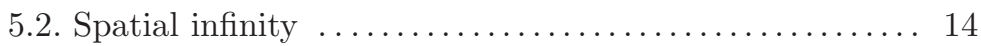

5.2.1. The electromagnetic twist potential and the norm of the axial Killing vector ...................... 15

5.2.2. The electromagnetic potentials $\ldots \ldots \ldots \ldots \ldots \ldots, 18$

6. Weinstein Solutions: existence and uniqueness ............ 19

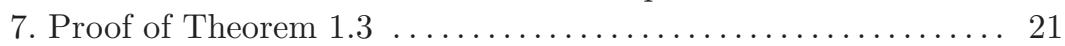

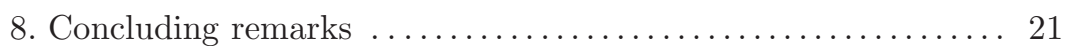

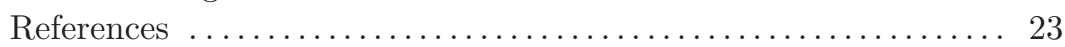

\section{Introduction}

We address the following celebrated and long-standing conjecture:

Conjecture 1.1. - Let $(\mathscr{M}, \mathfrak{g}, F)$ be a stationary, asymptotically flat, electrovacuum, four-dimensional regular space-time. Then the domain of outer communications $\left\langle\left\langle\mathscr{M}_{\mathrm{ext}}\right\rangle\right\rangle$ is either isometric to the domain of outer communications of a Kerr-Newman space-time or to the domain of outer communications of a (standard) Majumdar-Papapetrou space-time. 
Arguments to this effect have been given in the literature $[5,21,26]$ (see also [22, $36]$ ), with the hypotheses needed not always spelled out, and with some notable technical gaps. The aim of this work is to give continuation to the project initiated in [13], where the vacuum case was considered, and obtain a precise classification of such electro-vacuum solutions in the class of analytic space-times with nondegenerate event horizons, providing detailed filling of the gaps alluded to above.

As usual, in mathematical Relativity, part of the challenge posed by a conjecture is to obtain a precise formulation. In the case of the "no-hair" conjectures this non-linearity lies in the notion of regularity and it is our opinion that the nonexistence of a precise formalization for this concept has led to the enclosure of "hairy" assumptions and technical difficulties which has made the state of the art concerning this problem difficult to assess (1) So, we start exactly by collecting our technical assumptions in the following (we refer to Section 2 for the necessary intermediary definitions):

DEFINITION 1.2. - Let $(\mathscr{M}, \mathfrak{g})$ be a space-time containing an asymptotically flat end $\mathscr{S}_{\text {ext }}$, and let $K$ be a stationary Killing vector field on $\mathscr{M}$. We will say that $(\mathscr{M}, \mathfrak{g}, K)$ is $I^{+}{ }_{\text {-regular if } K}$ is complete, if the domain of outer communications $\left\langle\left\langle\mathscr{M}_{\text {ext }}\right\rangle\right\rangle$ is globally hyperbolic, and if $\left\langle\left\langle\mathscr{M}_{\text {ext }}\right\rangle\right\rangle$ contains a spacelike, connected, acausal hypersurface $\mathscr{S} \supset \mathscr{S}_{\text {ext }}$, the closure $\overline{\mathscr{S}}$ of which is a topological manifold with boundary, consisting of the union of a compact set and of a finite number of asymptotic ends, such that the boundary $\partial \overline{\mathscr{S}}:=\overline{\mathscr{S}} \backslash \mathscr{S}$ is a topological manifold satisfying

$$
\partial \overline{\mathscr{S}} \subset \mathscr{E}^{+}:=\partial\left\langle\left\langle\mathscr{M}_{\text {ext }}\right\rangle\right\rangle \cap I^{+}\left(\mathscr{M}_{\text {ext }}\right)
$$

with $\partial \overline{\mathscr{S}}$ meeting every generator of $\mathscr{E}^{+}$precisely once. (See Figure 1.1.)

Needless to say, all these conditions are satisfied by the Kerr-Newman and the Majumdar-Papapetrou solutions and, in particular, by Minkowski and ReissnerNordström. For a detailed discussion of the previous definition and an alternative formulation of Conjecture 1.1 with regular replaced by a specific set of weaker conditions see [13].

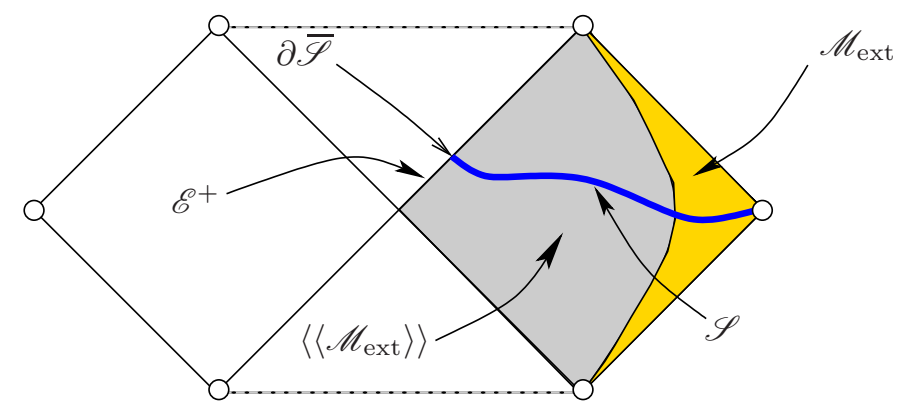

Figure 1.1. The hypersurface $\mathscr{S}$ from the definition of $I^{+}$-regularity.

(1) An illustrative example is given by the product structure 3.15 that, although clear for Minkowski with the usual $\mathbb{R} \times U(1)$ action by isometries, seems far from obvious in the generality required. Other examples are the regularity of the horizon and the asymptotic behavior of the relevant harmonic maps. 
In this work we establish the following special case of Conjecture 1.1

TheOrem 1.3. - Let $(\mathscr{M}, \mathfrak{g}, F)$ be a stationary, asymptotically flat, $I^{+}{ }_{-}$regular, electro-vacuum, four-dimensional analytic space-time, satisfying (2.7) and (2.11). If each component of the event horizon is mean non-degenerate, then $\left\langle\left\langle\mathscr{M}_{\mathrm{ext}}\right\rangle\right\rangle$ is isometric to the domain of outer communications of one of the Weinstein solutions of Section 6. In particular, if the event horizon is connected and mean nondegenerate, then $\left\langle\left\langle\mathscr{M}_{\mathrm{ext}}\right\rangle\right\rangle$ is isometric to the domain of outer communications of a Kerr-Newman space-time.

It should be emphasized that the hypotheses of analyticity and non-degeneracy are highly unsatisfactory, and one believes that they are not needed for the conclusion. Note that by not allowing the existence of the "technically awkward" [6] degenerate horizons we eliminate extreme Kerr-Newman as well as the MajumdarPapapetrou solutions from our classification. One also believes, in accordance with the statement of Conjecture 1.1, that all solutions with non-connected event horizon are in the Majumdar-Papapetrou family; consequently one expects all other (non-connected) Weinstein solutions, and in particular the ones referred to in the previous result, to be singular. We postpone further discussion of this issues to the final section.

A critical remark comparing our work with the existing literature is in order; we focus on those points that do not generalize immediately when passing from pure to electro-vacuum. First of all, the famous reduction of the Einstein-Maxwell source free equations to a singular harmonic map problem requires the use of Weyl coordinates. The local existence of such coordinates has been well known for some time now, but global existence has, to our knowledge, either been part of the ansatz, usually implicitly, or based on incorrect or incomplete analysis. The main reasons for this unsatisfactory situation resides in the existing proofs of non-negativity of the area function (3.10) in $\left\langle\left\langle\mathscr{M}_{\text {ext }}\right\rangle\right\rangle$, and existence of a global cross-section for the $\mathbb{R} \times U(1)$ action again in $\left\langle\left\langle\mathscr{M}_{\text {ext }}\right\rangle\right\rangle$. In [13] we solved this problems for vacuum and in Section 3 we present the necessary adjustments to extend this global existence result to the electro-vacuum scenario. Also, no previous work known to us establishes the asymptotic behavior, as needed for the proof of uniqueness, of the relevant harmonic maps. More specifically: the necessity to control the behavior at points where the horizon meets the rotation axis, prior to [13], seems to have been neglected; at infinity, which requires special attention in the electro-vacuum case, part of the necessary estimates were imposed as extra conditions, beyond asymptotic flatness; (2) also, an apparent disregard for the singular character, at the axis, of the hyperbolic distance (4.14) between the maps, even at large distance, appears to be the norm. A detailed asymptotic analysis is carried out in Section 5 .

We also note that a considerable part of the foundations of the theory underlying the desired classification depend exclusively on stationarity, $I^{+}$-regularity and the null energy condition. Again, this work was carried out in [13], where various results were established under conditions weaker than previously cited, or were generalized to higher dimensions; this is of potential interest for further work on the subject.

${ }^{(2)}$ See, for example, Theorem 2 in [27]. 


\section{Preliminaries}

An electro-vacuum space-time is a triple $(\mathscr{M}, \mathfrak{g}, F)$, assembled by a $(n+1)$ dimensional Lorentzian manifold $(\mathscr{M}, \mathfrak{g})$ endowed with a 2 -form $F$, that satisfies the source free Einstein-Maxwell field equations

$$
\begin{gathered}
\mathrm{Ric}-\frac{1}{2} \mathrm{Rg}=2 \mathrm{~T}_{F}, \\
F=d A, \\
d * F=0,
\end{gathered}
$$

where Ric is the Ricci curvature tensor of the metric $\mathfrak{g}, \mathrm{R}$ its scalar curvature and $\mathrm{T}_{F}$ is the energy-momentum tensor of the electromagnetic 2-form $F$,

$$
\mathrm{T}_{F}(u, v):=\mathfrak{g}\left(i_{u} F, i_{v} F\right)-\frac{1}{2}|F|^{2} \mathfrak{g}(u, v) .
$$

A space-time will be said to possess an asymptotically flat end if $\mathscr{M}$ contains a spacelike hypersurface $\mathscr{S}_{\text {ext }}$ diffeomorphic to $\mathbb{R}^{n} \backslash B(R)$, where $B(R)$ is an open coordinate ball of radius $R$, with the following properties: there exists a constant $\alpha>0$ such that, in local coordinates on $\mathscr{S}_{\text {ext }}$ obtained from $\mathbb{R}^{n} \backslash B(R)$, the metric $\gamma$ induced by $\mathfrak{g}$ on $\mathscr{S}_{\text {ext }}$, and the extrinsic curvature tensor $K_{i j}$ of $\mathscr{S}_{\text {ext }}$, satisfy the fall-off conditions

$$
\gamma_{i j}-\delta_{i j}=O_{k}\left(r^{-\alpha}\right), \quad K_{i j}=O_{k-1}\left(r^{-1-\alpha}\right),
$$

for some $k \geq 1$, where we write $f=O_{k}\left(r^{\alpha}\right)$ if $f$ satisfies

$$
\partial_{k_{1}} \ldots \partial_{k_{\ell}} f=O\left(r^{\alpha-\ell}\right), \quad 0 \leq \ell \leq k .
$$

In connection with the field equations we also request the following decay rate for the electromagnetic potential

$$
A_{\mu}=O_{k}\left(r^{-\alpha}\right) .
$$

A Killing vector $K$ is said to be complete if for every $p \in \mathscr{M}$ the orbit $\phi_{t}[K](p)$ of $K$ is defined for all $t \in \mathbb{R}$, i.e., if (the flow of) $K$ generates an action of $\mathbb{R}$ by isometries; in an asymptotically flat context, $K$ is called stationary if it is timelike at large distances. The exterior region $\mathscr{M}_{\text {ext }}$ and the domain of outer communications $\left\langle\left\langle\mathscr{M}_{\text {ext }}\right\rangle\right\rangle$ are then defined as

$$
\left\langle\left\langle\mathscr{M}_{\text {ext }}\right\rangle\right\rangle=I^{+}(\underbrace{\cup_{t} \phi_{t}\left(\mathscr{S}_{\text {ext }}\right)}_{=: \mathscr{M}_{\text {ext }}}) \cap I^{-}\left(\cup_{t} \phi_{t}\left(\mathscr{S}_{\text {ext }}\right)\right),
$$

with the event horizon being

$$
\mathscr{E}:=\partial\left\langle\left\langle\mathscr{M}_{\text {ext }}\right\rangle\right\rangle \quad ; \quad \mathscr{E}^{ \pm}:=I^{ \pm}\left(\mathscr{M}_{\text {ext }}\right) \cap \mathscr{E} .
$$

One expects stationary electro-vacuum space-times to be static or stationaryaxisymmetric: static meaning that the stationary Killing vector is hypersurfaceorthogonal, i.e.,

$$
d K^{\mathrm{b}} \wedge K^{\mathrm{b}}=0,
$$

where $K^{b}=\mathfrak{g}(K, \cdot)$, and stationary-axisymmetric corresponding to the existence of a second complete Killing vector $K_{(1)}$, which together with the stationary Killing vector $K_{(0)}:=K$ generate an $\mathbb{R} \times \mathbf{U}(1)$ action by isometries. In connection with 
the field equations we require the electromagnetic field to be invariant under the flow of the relevant Killing vectors

$$
\mathscr{L}_{K_{(\mu)}} F=0
$$

In the stationary and asymptotic flat scenario one is able to choose adapted coordinates so that the metric can, in a neighborhood of infinity, be written as

$$
\mathfrak{g}=-V^{2}(d t+\underbrace{\theta_{i} d x^{i}}_{=\theta})^{2}+\underbrace{\gamma_{i j} d x^{i} d x^{j}}_{=\gamma},
$$

with

$$
K=\partial_{t} \Longrightarrow \partial_{t} V=\partial_{t} \theta_{i}=\partial_{t} \gamma_{i j}=0 ;
$$

since we are also assuming electro-vacuum we get the following improvement of the original decay rates [7, Section 1.3],

$$
\gamma_{i j}-\delta_{i j}=O_{\infty}\left(r^{-1}\right), \quad \theta_{i}=O_{\infty}\left(r^{-1}\right), \quad V-1=O_{\infty}\left(r^{-1}\right),
$$

and

$$
A_{\mu}=O_{\infty}\left(r^{-1}\right),
$$

where the infinity symbol means that (2.6) holds for arbitrary $k$.

\section{Weyl coordinates}

On a region charted by Weyl coordinates the source free Einstein-Maxwell equations simplify considerably. It has been for long expected and recently showed in [13] that such global chart is available away from the axis of a stationary and axisymmetric vacuum domain of outer communications. In fact the role of the vacuum field equations in the referred analysis - they imply the orthogonal integrability conditions (3.1) and allow us to show that, whenever defined, the squared root of the area function (3.10) is harmonic with respect to the orbit space metric - is fulfilled by the electro-vacuum field equations.

The first of these well known results, which neither requires $K_{(0)}$ to be stationary, nor $K_{(1)}$ to be a generator of axisymmetry, generalizes to higher dimensions as follows (compare [4]):

Proposition 3.1. - Let $(\mathscr{M}, \mathfrak{g}, F)$ be an $(n+1)$-dimensional electro-vacuum spacetime, possibly with a cosmological constant, with $n-1$ commuting Killing vector fields satisfying

$$
\mathscr{L}_{K_{(\mu)}} F=0, \mu=0, \ldots, n-2 .
$$

If $n-2$ of the zero sets $\mathscr{A}_{\mu}:=\left\{p \in \mathscr{M}\left|K_{(\mu)}\right|_{p}=0\right\}$ are non-empty then (3)

$$
d K_{(\mu)} \wedge K_{(0)} \wedge \ldots \wedge K_{(n-2)}=0 \quad, \forall \mu=0, \ldots, n-2 .
$$

Proof. - To fix conventions, we use a Hodge star defined through the formula

$$
\alpha \wedge \beta= \pm\langle * \alpha, \beta\rangle \mathrm{Vol},
$$

where the plus sign is taken in the Riemannian case, minus in our Lorentzian one, while $\mathrm{Vol}$ is the volume form. The following (well known) identities are useful [22];

$$
* * \theta=(-1)^{s(n+1-s)-1} \theta, \quad \forall \theta \in \Lambda^{s},
$$

${ }^{(3)} \mathrm{By}$ an abuse of notation, we use the same symbols for vector fields and for the associated 1-forms. 


$$
i_{X} * \theta=*(\theta \wedge X), \quad \forall \theta \in \Lambda^{s}, \quad X \in \Lambda^{1} .
$$

Further, for any Killing vector $K$,

$$
\left[\mathscr{L}_{K}, *\right]=0 .
$$

The Leibniz rule for the divergence $\delta:=* d *$ reads, for $\theta \in \Lambda^{s}$,

$$
\begin{aligned}
\delta(\theta \wedge K) & =* d *(\theta \wedge K) \stackrel{\sqrt{3.36}}{=} * d\left(i_{K} * \theta\right)=*\left(\mathscr{L}_{K} * \theta-i_{K} d * \theta\right) \\
& \stackrel{\text { [.2. (3.4) }}{=} * * \mathscr{L}_{K} \theta-* i_{K}(-1)^{(n+1-s+1)(n+1-(n+1-s+1))-1} * * d * \theta \\
& =(-1)^{s(n+1-s)-1} \mathscr{L}_{K} \theta-(-1)^{s(n+1-s)-n+1} * *(\delta \theta \wedge K) \\
& =(-1)^{s(n+1-s)-1} \mathscr{L}_{K} \theta+(-1)^{n+1} \delta \theta \wedge K .
\end{aligned}
$$

Applying this to $\theta=d K$ one obtains

$$
\begin{aligned}
* d *(d K \wedge K) & =-\mathscr{L}_{K} d K+(-1)^{n+1} \delta d K \wedge K \\
& =(-1)^{n+1} \delta d K \wedge K .
\end{aligned}
$$

As any Killing vector is divergence free, we see that

$$
\delta d K=(-1)^{n} \Delta K=(-1)^{n} 2 \operatorname{tr} \nabla^{2} K=(-1)^{n+1} 2 i_{K} \text { Ric },
$$

where $\Delta$ is the Laplace-Beltrami operator. The assumed field equations (with cosmological constant $\Lambda$ ) imply

$$
\text { Ric }=2 \mathrm{~T}_{F}+\frac{2}{n-1} \Lambda \mathfrak{g}
$$

from which

$$
\begin{aligned}
* d *(d K \wedge K) & =(-1)^{n+1}(-1)^{n+1} 2 i_{K}\left(2 \mathrm{~T}_{F}+\frac{2}{n-1} \Lambda \mathfrak{g}\right) \wedge K \\
& =2\left(2 i_{K} \mathrm{~T}_{F} \wedge K+\frac{2}{n-1} \Lambda K\right) \wedge K=4 i_{K} \mathrm{~T}_{F} \wedge K .
\end{aligned}
$$

Letting $\alpha:=i_{K} F$ for any vector field $X$ we have

$$
\begin{aligned}
\alpha \cdot i_{X} F & =-*\left(\alpha \wedge * i_{X} F\right)=-(-1)^{n} *\left(* i_{X} F \wedge \alpha\right) \\
& =(-1)^{n+1} i_{\alpha} * * F=(-1)^{n+1}(-1)^{n+1} i_{\alpha} i_{X} F \\
& =-F(\alpha, X)
\end{aligned}
$$

which inserted into (2.4) gives

$$
i_{K} T_{F}=-i_{\alpha} F-\frac{1}{2}|F|^{2} K
$$

and consequently

$$
* d *(d K \wedge K)=-4\left(i_{\alpha} F+\frac{1}{2}|F|^{2} K\right) \wedge K=-4 i_{\alpha} F \wedge K=4 K \wedge i_{\alpha} F .
$$

Meanwhile, since (modulo sign)

$$
\begin{aligned}
i_{\alpha} K & = \pm *(K \wedge * \alpha)= \pm *\left(K \wedge * i_{K} F\right)= \pm *\left(K \wedge * i_{K} * * F\right) \\
& = \pm *(K \wedge * *(* F \wedge K))= \pm *(K \wedge * F \wedge K)=0
\end{aligned}
$$


for $\beta:=i_{K} * F \in \Lambda^{n-2}$, we have

$$
\begin{aligned}
*(\alpha \wedge \beta) & =(-1)^{1 \times(n-2)} *(\beta \wedge \alpha)=(-1)^{n-2} i_{\alpha} * \beta \\
& =(-1)^{n-2} i_{\alpha} * i_{K} * F=(-1)^{n-2} i_{\alpha} * *(F \wedge K) \\
& =(-1)^{n-2} i_{\alpha}(-1)^{3(n+1-3)-1} F \wedge K \\
& =-\left(i_{\alpha} F \wedge K+(-1)^{2} F \wedge i_{\alpha} K\right)=-\left(i_{\alpha} F \wedge K+0\right) \\
& =K \wedge i_{\alpha} F
\end{aligned}
$$

which leads to the significant

$$
d *(d K \wedge K)=4 \alpha \wedge \beta=4 i_{K} F \wedge i_{K} * F .
$$

Now, for any two commuting Killing vectors and an arbitrary differential form we have

$$
\begin{aligned}
{\left[\mathscr{L}_{K_{(\mu)}}, i_{K_{(\nu)}}\right] \theta } & =\mathscr{L}_{K_{(\mu)}}\left(i_{K_{(\nu)}} \theta\right)-i_{K_{(\nu)}}\left(\mathscr{L}_{K_{(\mu)}} \theta\right) \\
& =\mathscr{L}_{K_{(\mu)}}\left[\theta\left(K_{(\nu)}, \ldots\right)\right]-\left(\mathscr{L}_{K_{(\mu)}} \theta\right)\left(K_{(\nu)}, \ldots\right) \\
& =\left(\mathscr{L}_{K_{(\mu)}} \theta\right)\left(K_{(\nu)}, \ldots\right)+\theta\left(\mathscr{L}_{K_{(\mu)}} K_{(\nu)}, \ldots\right)-\left(\mathscr{L}_{K_{(\mu)}} \theta\right)\left(K_{(\nu)}, \ldots\right)=0
\end{aligned}
$$

giving us the commutation relation

$$
\left[K_{(\mu)}, K_{(\nu)}\right]=0 \Longrightarrow\left[\mathscr{L}_{K_{(\mu)}}, i_{K_{(\nu)}}\right]=0,
$$

from which it follows that

$$
\begin{aligned}
d F\left(K_{(\nu)}, K_{(\mu)}\right) & =d i_{K_{(\mu)}} \alpha_{(\nu)}=-i_{K_{(\mu)}} d \alpha_{(\nu)}+\mathscr{L}_{K_{(\mu)}} \alpha_{(\mu)} \\
& =-i_{K_{(\mu)}}\left(-i_{K_{(\nu)}} d F+\mathscr{L}_{K_{(\nu)}} F\right)+i_{K_{(\mu)}} \mathscr{L}_{K_{(\mu)}} F=0,
\end{aligned}
$$

where we used the fact that $F$ is exact and invariant under the flow of this Killing vectors. By the hypothesis on the zero sets, for any pair $\mu \neq \nu$, we may take $\mathscr{A}_{(\mu)} \neq \emptyset$. We then have $\left.F\left(K_{(\mu)}, K_{(\nu)}\right)\right|_{\mathscr{A}_{(\mu)}} \equiv 0$ and consequently

$$
F\left(K_{(\mu)}, K_{(\nu)}\right) \equiv 0, \forall \mu, \nu \in\{0, \ldots, n-2\} .
$$

A similar computation leads to

$$
i_{K_{(\mu)}} i_{K_{(\nu)}} * F=0, \forall \mu, \nu \in\{0, \ldots, n-2\} .
$$

Now, let $\omega_{(\mu)}$ be the $\mu$ 'th twist form,

$$
\omega_{(\mu)}:=*\left(d K_{(\mu)} \wedge K_{(\mu)}\right) .
$$

The identity

$$
\begin{aligned}
\mathscr{L}_{K_{(\mu)}} \omega_{(\nu)} & =\mathscr{L}_{K_{(\mu)}} *\left(d K_{(\mu)} \wedge K_{(\nu)}\right) \\
& =*\left(\mathscr{L}_{K_{(\mu)}} d K_{(\nu)}+d K_{(\nu)} \wedge \mathscr{L}_{K_{(\mu)}} K_{(\nu)}\right)=0,
\end{aligned}
$$

together with

$$
\mathscr{L}_{K_{\left(\mu_{1}\right)}}\left(i_{K_{\left(\mu_{2}\right)}} \ldots i_{K_{\left(\mu_{\ell}\right)}} \omega_{\left(\mu_{\ell+1}\right)}\right)=i_{K_{\left(\mu_{2}\right)}} \ldots i_{K_{\left(\mu_{n-1}\right)}} \mathscr{L}_{K_{\left(\mu_{\ell}\right)}} \omega_{\left(\mu_{\ell+1}\right)}=0,
$$

and Cartan's formula for the Lie derivative, gives

$$
d\left(i_{K_{\left(\mu_{1}\right)}} \ldots i_{K_{\left(\mu_{\ell}\right)}} \omega_{\left(\mu_{\ell+1}\right)}\right)=(-1)^{\ell} i_{K_{\left(\mu_{1}\right)}} \ldots i_{K_{\left(\mu_{n-1}\right)}} d \omega_{\left(\mu_{\ell+1}\right)} .
$$


We thus have

$$
\begin{aligned}
& d *\left(d K_{\left(\mu_{0}\right)} \wedge K_{\left(\mu_{0}\right)} \wedge \ldots \wedge K_{\left(\mu_{n-2}\right)}\right)=d\left(i_{K_{\left(\mu_{n-2}\right)}} \ldots i_{K_{\left(\mu_{1}\right)}} *\left(d K_{\left(\mu_{0}\right)} \wedge K_{\left(\mu_{0}\right)}\right)\right) \\
& =(-1)^{n-2} i_{K_{\left(\mu_{n-2}\right)}} \ldots i_{K_{\left(\mu_{1}\right)}} d \omega_{\left(\mu_{0}\right)} \\
& \stackrel{\sqrt[3.5]{=}}{=}(-1)^{n} i_{K_{\left(\mu_{n-2}\right)}} \cdots i_{K_{\left(\mu_{1}\right)}} 4 \alpha_{\left(\mu_{0}\right)} \wedge \beta_{\left(\mu_{0}\right)} \\
& =4(-1)^{n} i_{K_{\left(\mu_{n-2}\right)}} \ldots i_{K_{\left(\mu_{2}\right)}} \\
& \left(i_{K_{\left(\mu_{1}\right)}} \alpha_{\left(\mu_{0}\right)} \wedge \beta_{\left(\mu_{0}\right)}-\alpha_{\left(\mu_{0}\right)} \wedge i_{K_{\left(\mu_{1}\right)}} \beta_{\left(\mu_{0}\right)}\right) \\
& =4(-1)^{n} i_{K_{\left(\mu_{n-2}\right)}} \ldots i_{K_{\left(\mu_{2}\right)}} \\
& \left(F\left(K_{\left(\mu_{0}\right)}, K_{\left(\mu_{1}\right)}\right) \beta_{\left(\mu_{0}\right)}-\alpha_{\left(\mu_{0}\right)} \wedge i_{K_{\left(\mu_{1}\right)}} i_{K_{\left(\mu_{0}\right)}} * F\right) \\
& \stackrel{3.713 .8}{=} 0 \text {. }
\end{aligned}
$$

So the function $*\left(d K_{\left(\mu_{0}\right)} \wedge K_{\left(\mu_{0}\right)} \wedge K_{\left(\mu_{1}\right)} \wedge \ldots \wedge K_{\left(\mu_{n-2}\right)}\right)$ is constant, and, as before, the result follows from the hypothesis on the zero sets.

Noting that a globally hyperbolic, stationary and asymptotically flat domain of outer communications satisfying the null energy condition is necessarily simplyconnected $[16,19,20]$, in view of the previous result Theorem 5.6 of [13] translates to the electro-vacuum setting as:

Theorem 3.2. - Let $(\mathscr{M}, \mathfrak{g}, F)$ be a four-dimensional, $I^{+}$-regular, asymptotically flat, electro-vacuum space-time with stationary Killing vector $K_{(0)}$ and periodic Killing vector $K_{(1)}$, jointly generating an $\mathbb{R} \times \mathrm{U}(1)$ subgroup of the isometry group of $(\mathscr{M}, \mathfrak{g})$. If $\left\langle\left\langle\mathscr{M}_{\text {ext }}\right\rangle\right\rangle$ is globally hyperbolic, then the area function

$$
W:=-\operatorname{det}\left(\mathfrak{g}\left(K_{(\mu)}, K_{(\nu)}\right)\right)_{\mu, \nu=0,1},
$$

is non-negative on $\left\langle\left\langle\mathscr{M}_{\mathrm{ext}}\right\rangle\right\rangle$, vanishing precisely on the union of its boundary with the (non-empty) set $\left\{\mathfrak{g}\left(K_{(1)}, K_{(1)}\right)=0\right\}$.

Away from points where $K_{(0)} \wedge K_{(1)}$ vanishes, which according to [13, Corollary $3.8]$ correspond, in a chronologica $\left\langle(4)\left\langle\left\langle\mathscr{M}_{\text {ext }}\right\rangle\right\rangle\right.$, exactly to axis points

$$
\mathscr{A}:=\left\{q \in \mathscr{M}\left|K_{(1)}\right|_{q}=0\right\},
$$

there is a well defined and differentiable local cross-section for the $\mathbb{R} \times U(1)$ action. We can endow this cross-section with the orbit space metric

$$
q\left(Z_{1}, Z_{2}\right)=\mathfrak{g}\left(Z_{1}, Z_{2}\right)-h^{\mu \nu} \mathfrak{g}\left(Z_{1}, K_{(\mu)}\right) \mathfrak{g}\left(Z_{2}, K_{(\nu)}\right),
$$

whenever $h_{\mu \nu}:=\mathfrak{g}\left(K_{(\mu)}, K_{(\nu)}\right)$ is non-singular. The established orthogonality conditions allow us to identify, at least locally, the previous orbit space structure with a 2-surface orthogonal to the Killing vectors, provided by (3.1), endowed with the induced metric. From this and Theorem (3.2) we see that $q$ is well defined and Riemannian throughout $\left\langle\left\langle\mathscr{M}_{\text {ext }}\right\rangle\right\rangle \backslash \mathscr{A}$; it is then well known [36] that

$$
\Delta_{q} \sqrt{W}=0
$$

whenever $W$ is non-negative and $q$ is Riemannian, which again is the case within $\langle\langle\mathscr{M}$ ext $\rangle\rangle \backslash \mathscr{A}$.

(4) No closed timelike curves allowed. 
According to the Structure Theorem [13], $I^{+}$-regularity allows for the decomposition

$$
\overline{\left\langle\left\langle\mathscr{M}_{\mathrm{ext}}\right\rangle\right\rangle} \cap I^{+}\left(\mathscr{M}_{\mathrm{ext}}\right)=\mathbb{R} \times \overline{\mathscr{S}},
$$

with $K_{(1)}$ tangent to $\overline{\mathscr{S}}$, a simply-connected spacelike hypersurface with boundary which is an asymptotically flat global cross-section for the action generated by the stationary vector. We are now allowed to use the classification of circle actions on simply-connected 3-manifolds of Orlik and Raymond [29,31] to obtain a global cross-section for the $\mathbb{R} \times \mathbf{U}(1)$ action in $\left\langle\left\langle\mathscr{M}_{\text {ext }}\right\rangle\right\rangle \backslash \mathscr{A}$. Then, by (3.13) and relying on the results of [10], while disallowing the existence of degenerate horizons, we are able to undertake an analysis leading to

$$
\left\langle\left\langle\mathscr{M}_{\text {ext }}\right\rangle\right\rangle \backslash \mathscr{A} \approx \mathbb{R} \times S^{1} \times \mathbb{R}^{+} \times \mathbb{R},
$$

while showing that this diffeomorphism defines a global coordinate system $(t, \varphi, \rho, z)$ with

$$
K_{(0)}=\partial_{t}, \quad K_{(1)}=\partial_{\varphi} \text { and } \rho=\sqrt{W} .
$$

After invoking (3.1) once more, the desired global expression for the space-time metric in terms of Weyl coordinates

$$
\mathfrak{g}=-\rho^{2} e^{2 \lambda} d t^{2}+e^{-2 \lambda}(d \varphi-w d t)^{2}+e^{2 u}\left(d \rho^{2}+d z^{2}\right),
$$

follows, with

$$
u=O_{k-4}\left(r^{-1}\right), r=\sqrt{\rho^{2}+z^{2}} \rightarrow \infty .
$$

\section{Reduction to a harmonic map problem}

The electro-vacuum field equations (2.1)-(2.3) and simple-connectedness of $\left\langle\left\langle\mathscr{M}_{\text {ext }}\right\rangle\right\rangle$ guarantee the global existence of the following potentials:

$$
d \chi=i_{K_{(1)}} F \quad, \quad d \psi=i_{K_{(1)}} * F \quad \text { and } \quad d v=\omega-2(\chi d \psi-\psi d \chi),
$$

where

$$
\omega:=*\left(d K_{(1)}^{b} \wedge K_{(1)}^{b}\right),
$$

is the axial twist form. As discussed in detail in [36], when a global representation in terms of Weyl coordinates like (3.17) is allowed, the space-time metric is uniquely determined by an axisymmetric harmonic map

$$
\Phi=(\lambda, v, \chi, \psi): \mathbb{R}^{3} \backslash \mathscr{A} \longrightarrow \mathbb{H}_{\mathbb{C}}^{2},
$$

here $\mathscr{A}=\{(0,0, z) \mid z \in \mathbb{R}\}$ and $\mathbb{H}_{\mathbb{C}}^{2}$ is the 'upper half-space model' of the 2dimensional complex hyperbolic space, i.e., $\mathbb{R}^{4}$ with metric given by

$$
d s^{2}=d \lambda^{2}+e^{4 \lambda}(d v+\chi d \psi-\psi d \chi)^{2}+e^{2 \lambda}\left(d \chi^{2}+d \psi^{2}\right) .
$$

The metric coefficient $\lambda$ is part of the harmonic map and the remaining unknowns of the metric can be determined from $\Phi$ by considering the unique solution $(w, u)$ of the set of equations

$\partial_{\rho} u-\partial_{\rho} \lambda=\rho\left[\left(\partial_{\rho} \lambda\right)^{2}-\left(\partial_{z} \lambda\right)^{2}+\frac{1}{4} e^{4 \lambda}\left(\omega_{\rho}^{2}-\omega_{z}^{2}\right)+e^{2 \lambda}\left(\left(\partial_{\rho} \chi\right)^{2}-\left(\partial_{z} \chi\right)^{2}+\left(\partial_{\rho} \psi\right)^{2}-\left(\partial_{z} \psi\right)^{2}\right)\right]$

$$
\partial_{z} u-\partial_{z} \lambda=2 \rho\left[\partial_{\rho} \lambda \partial_{z} \lambda+\frac{1}{4} e^{4 \lambda} \omega_{\rho} \omega_{z}+e^{2 \lambda}\left(\partial_{\rho} \chi \partial_{z} \chi+\partial_{\rho} \psi \partial_{z} \psi\right)\right]
$$


that go to zero at infinity, and where we write $\omega_{a}:=\omega\left(\partial_{a}\right)$ for $a \in\{\rho, z\}$.

4.1. Distance function on the target manifold. - The criteria for uniqueness of harmonic maps used in this paper (see Theorem 6.1 and compare [12, Appendix $\mathrm{C}]$ ), is stated in terms of the pointwise distance between the maps. For the 'disk model' of $\mathbb{H}_{\mathbb{C}}^{2}$ the distance between two points $z=\left(z_{1}, z_{2}\right)$ and $w=\left(w_{1}, w_{2}\right)$ is given by $[35$, eq 55 , pg 26$]$

$$
\cosh (d)=\frac{\left|1-\bar{z}_{1} w_{1}-\bar{z}_{2} w_{2}\right|}{\sqrt{1-|z|^{2}} \sqrt{1-|w|^{2}}} .
$$

To obtain the distance function for the 'upper half-space model' we will use the isometry between the two referred models presented in [35, Appendix]: first we perform the coordinate transformation

$$
z_{1}=\frac{1-x_{1}}{1+x_{1}} \quad, \quad z_{2}=\frac{2 x_{2}}{1+x_{1}}
$$

with analogous expressions for $w_{i}=w_{i}\left(y_{1}, y_{2}\right)$ to obtain

$$
\left|1-\bar{z}_{1} w_{1}-\bar{z}_{2} w_{2}\right|=\frac{2\left|\bar{x}_{1}+y_{1}-2 \bar{x}_{2} y_{2}\right|}{\left|1+x_{1}\right|\left|1+y_{1}\right|} ;
$$

then we take

so that

$$
e^{\lambda_{1}}=\frac{\left|1+z_{1}\right|}{\sqrt{1-|z|^{2}}} \quad \text { and } \quad e^{\lambda_{2}}=\frac{\left|1+h_{1}\right|}{\sqrt{1-|h|^{2}}}
$$

$$
\cosh (d)=\frac{1}{2}\left|\bar{x}_{1}+y_{1}-2 \bar{x}_{2} y_{2}\right| e^{\lambda_{1}+\lambda_{2}} ;
$$

and finally, by writing

$$
x_{1}=e^{-2 \lambda_{1}}+\chi_{1}^{2}+\psi_{1}^{2}+2 i v_{1} \quad \text { and } \quad x_{2}=\chi_{1}+i \psi_{1},
$$

with similar expressions for $y_{i}=y_{i}\left(\lambda_{2}, v_{2}, \chi_{2}, \psi_{2}\right)$, we see that the distance function satisfies (5)

$$
\begin{aligned}
\cosh ^{2}(d) & =\frac{1}{4} e^{2\left(\lambda_{1}+\lambda_{2}\right)}\left(e^{-2 \lambda_{1}}+e^{-2 \lambda_{2}}+\left(\chi_{1}-\chi_{2}\right)^{2}+\left(\psi_{1}-\psi_{2}\right)^{2}\right)^{2} \\
& +e^{2\left(\lambda_{1}+\lambda_{2}\right)}\left(v_{2}-v_{1}-\chi_{1} \psi_{2}+\chi_{2} \psi_{1}\right)^{2} \\
& =\frac{1}{4}\left\{e^{-\lambda_{1}+\lambda_{2}}+e^{\lambda_{1}-\lambda_{2}}+e^{\lambda_{1}+\lambda_{2}}\left(\chi_{1}-\chi_{2}\right)^{2}+e^{\lambda_{1}+\lambda_{2}}\left(\psi_{1}-\psi_{2}\right)^{2}\right\}^{2} \\
& +e^{2\left(\lambda_{1}+\lambda_{2}\right)}\left\{\left(v_{2}-v_{1}\right)+\left(\chi_{2} \psi_{1}-\chi_{1} \psi_{2}\right)\right\}^{2},
\end{aligned}
$$

or in an apparently more intrinsic way

$$
\begin{aligned}
\cosh ^{2}(d) & =\frac{1}{4}\left\{\sqrt{\frac{\mathfrak{g}_{2}\left(\partial_{\varphi}, \partial_{\varphi}\right)}{\mathfrak{g}_{1}\left(\partial_{\varphi}, \partial_{\varphi}\right)}}+\sqrt{\frac{\mathfrak{g}_{1}\left(\partial_{\varphi}, \partial_{\varphi}\right)}{\mathfrak{g}_{2}\left(\partial_{\varphi}, \partial_{\varphi}\right)}}+\frac{\left(\chi_{1}-\chi_{2}\right)^{2}+\left(\psi_{1}-\psi_{2}\right)^{2}}{\sqrt{\mathfrak{g}_{1}\left(\partial_{\varphi}, \partial_{\varphi}\right)} \sqrt{\mathfrak{g}_{2}\left(\partial_{\varphi}, \partial_{\varphi}\right)}}\right\}^{2} \\
& +\left\{\frac{\left(v_{2}-v_{1}\right)+\left(\chi_{2} \psi_{1}-\chi_{1} \psi_{2}\right)}{\sqrt{\mathfrak{g}_{1}\left(\partial_{\varphi}, \partial_{\varphi}\right)} \sqrt{\mathfrak{g}_{2}\left(\partial_{\varphi}, \partial_{\varphi}\right)}}\right\}^{2} .
\end{aligned}
$$

$\overline{\text { (5) By taking } \chi_{i}}=\psi_{i} \equiv 0$ we see that this distance function is related to the one used in the vacuum case [13, Section 6.5.1] by $d=2 d_{b}$. This discrepancy has its genesis in an analogous relation between the line elements of the different disk models used. 


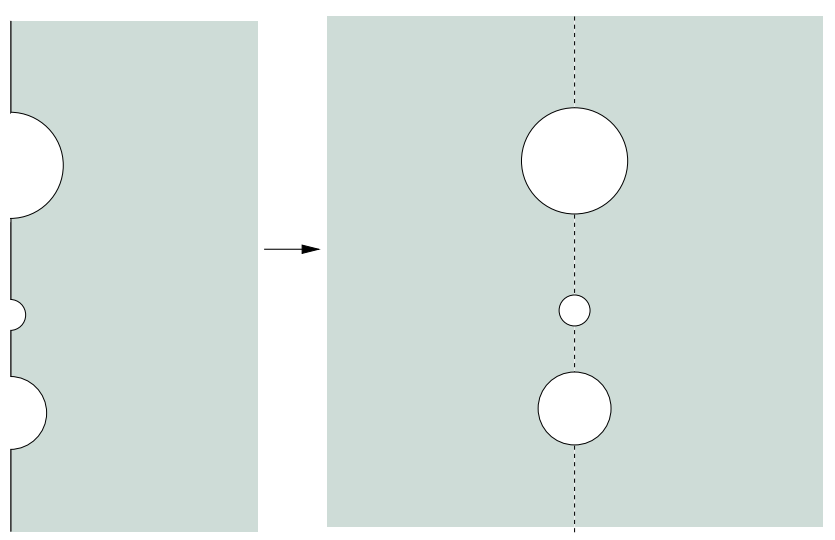

Figure 5.1. The quotient space and its double.

It will also be helpful to use the usual rescaling

$$
U_{i}=\lambda_{i}+\ln \rho, \text { so that } \mathfrak{g}_{i}\left(\partial_{\varphi}, \partial_{\varphi}\right)=\rho^{2} e^{-2 U_{i}}=e^{-2 \lambda_{i}},
$$

from which we get our final expression for the distance in the 'upper half-space':

$$
\begin{aligned}
\cosh ^{2}(d)= & \frac{1}{4}\left\{e^{U_{1}-U_{2}}+e^{-U_{1}+U_{2}}+\rho^{-2} e^{U_{1}+U_{2}}\left(\chi_{1}-\chi_{2}\right)^{2}+\rho^{-2} e^{U_{1}+U_{2}}\left(\psi_{1}-\psi_{2}\right)^{2}\right\}^{2} \\
& +\left\{\rho^{-2} e^{U_{1}+U_{2}}\left(v_{2}-v_{1}\right)-\rho^{-2} e^{U_{1}+U_{2}}\left(\chi_{1} \psi_{2}-\chi_{2} \psi_{1}\right)\right\}^{2}
\end{aligned}
$$

\section{Boundary conditions}

5.1. The Axis. - From now on we will be controlling the distance, as given by any of the formulae in the previous section, between the harmonic maps arising from two $I^{+}$-regular, stationary-axisymmetric and electro-vacuum space-times $\left(\mathscr{M}_{i}, \mathfrak{g}_{i}\right)$, $i=1,2$. We will start by showing that

$$
d\left(\Phi_{1}, \Phi_{2}\right) \text { is bounded near } \overline{\mathscr{A} \cap\left\langle\left\langle\mathscr{M}_{\text {ext }}\right\rangle\right\rangle} \text {. }
$$

In this section we will be working with the following coordinate systems: isothermal coordinates $\left(\hat{x}_{i}, \hat{z}_{i}\right)$ globally defined in the doubling across the axis of the orbit space of an appropriate extension of the $\mathbf{U}(1)$ action to the manifold obtained by the addition of 3-discs to every connected component of $\partial \mathscr{S}_{i},(6)$ "canonical coordinates" $(\rho, z)$ of the half plane $\mathbb{R}_{0}^{+} \times \mathbb{R}$, which is the image of each (physical) orbit space by the map $\Psi_{i}$ defined by $\left(\hat{x}_{i}, \hat{z}_{i}\right) \mapsto\left(\rho_{i}\left(\hat{x}_{i}, \hat{z}_{i}\right), z_{i}\left(\hat{x}_{i}, \hat{z}_{i}\right)\right)$.

Let $\phi_{s}$ be the flow generated by the axial Killing vector $K_{(1)}$. In the doubling of the orbit space the isothermal coordinates satisfy

$$
\hat{x} \circ \phi_{\pi}=-\hat{x} \quad \text { and } \quad \hat{z} \circ \phi_{\pi}=\hat{z} .
$$

Then, invariance of a function $(\hat{x}, \hat{z}) \mapsto f(\hat{x}, \hat{z})$ under the axial flow, which is the case for the fields $v, \chi$ and $\psi$, implies that the function $\hat{x} \mapsto f(\hat{x}, \hat{z})$ is even for all

${ }^{(6)}$ The resulting space is diffeomorphic to $\mathbb{R}^{2}$, see Figure 5.1 and for more details concerning this construction see [13, Section 6]; also, the fact that $I^{+}$-regularity, stationarity and the null energy condition imply spherical topology for the connected components of the cross-section of the event horizon $\partial \mathscr{S}_{i}$ follows from [16]. 
$\hat{z}$. In this case, if $f$ is $C^{2}$, Taylor expanding on $\hat{x}$, from the axis, gives

$$
f(\hat{x}, \hat{z})=f(0, \hat{z})+\frac{1}{2} \frac{\partial^{2} f}{\partial \hat{x}^{2}}(c(\hat{x}), \hat{z}) \hat{x}^{2},|c(\hat{x})| \leq|\hat{x}| .
$$

Now fix a point in $\overline{\mathscr{A} \cap\left\langle\left\langle\mathscr{M}_{\text {ext }}\right\rangle\right\rangle}$ and by rescaling $\hat{z}$ assume it lies at the origin. Sup-

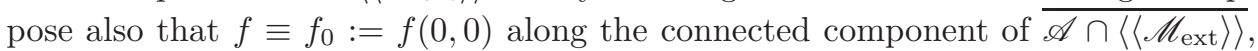
in $\overline{\left\langle\left\langle\mathscr{M}_{\text {ext }}\right\rangle\right\rangle}$, containing $(0,0)$; this is clearly the case for all the functions appearing in 4.11 and it also implies that we can realize the aforementioned extension of the doubling of the orbit space to $\mathbb{R}^{2}$ while preserving the constancy of $f$ along the extended axis near the poles, i.e., near the points where the axis meets the event horizon. Then (5.2) implies

$$
\left|f(\hat{x}, \hat{z})-f_{0}\right| \leq C \hat{x}^{2} \quad \text { near }(0,0) \in \overline{\mathscr{A} \cap\left\langle\left\langle\mathscr{M}_{\text {ext }}\right\rangle\right\rangle} .
$$

We will need bigger control over the functions $e^{-2 \lambda}=\mathfrak{g}_{\varphi \varphi}$. To this end let $\{x, y, z\}$ be Gaussian coordinates along the axis, in the extension of $\mathscr{S}$, with $\mathscr{A}=\{x=y=$ $0\}$ and for which $K_{(1)}=x \partial_{y}-y \partial_{x}$ (see [10,pg 5] and compare with (5.23) ). For any path with initial velocity transverse to $\mathscr{A}$ we have

$$
\left.\nabla_{\dot{\gamma}(0)} K_{(1)}\right|_{x=y=0}=\left.\nabla_{\gamma^{i} \partial_{i}}\left(x \partial_{y}-y \partial_{x}\right)\right|_{x=y=0}=\gamma^{x} \partial_{y}-\gamma^{y} \partial_{x},
$$

and consequently $\mathfrak{g}\left(\nabla_{\dot{\gamma}(0)} K_{(1)}, \nabla_{\dot{\gamma}(0)} K_{(1)}\right)=\left(\gamma^{x}\right)^{2}+\left(\gamma^{y}\right)^{2} \neq 0$. Since $\nabla_{\mu} \mathfrak{g}\left(K_{(1)}, K_{(1)}\right)=$ $2 \mathfrak{g}\left(\nabla_{\mu} K_{(1)}, K_{(1)}\right)$ we see that the gradient of $\mathfrak{g}_{\varphi \varphi}$ vanishes at the axis and

$$
\left.\nabla_{\mu} \nabla_{\nu} \mathfrak{g}\left(K_{(1)}, K_{(1)}\right)\right|_{\mathscr{A}}=\left.2 \mathfrak{g}\left(\nabla_{\mu} K_{(1)}, \nabla_{\nu} K_{(1)}\right)\right|_{\mathscr{A}} .
$$

Taylor expanding along $\gamma$ yields

$$
\mathfrak{g}_{\varphi \varphi} \circ \gamma(s)=(\underbrace{\mathfrak{g}\left(\nabla_{\dot{\gamma}(0)} K_{(1)}, \nabla_{\dot{\gamma}(0)} K_{(1)}\right)}_{\neq 0}+O(s)) s^{2},
$$

from which it follows that for any path transverse to $\mathscr{A}$ and small $s$

$$
C^{-1} s^{2} \leq \mathfrak{g}_{\varphi \varphi} \circ \gamma(s) \leq C s^{2} .
$$

We will need to consider two separate cases. First, fix, in each space-time, a point belonging to $\mathscr{A}_{i} \cap\left\langle\left\langle\mathscr{M}_{\text {ext }}\right\rangle\right\rangle$ and rescale all the previous coordinate systems so that each of the fixed points corresponds to its respective origin and $\Psi_{i}(0,0)=$ $(0,0)$. At these points, since there the boundary of the orbit space is analytic, the function $\rho_{i}=\rho_{i}\left(\hat{x}_{i}, \hat{z}_{i}\right)$ may be extended analytically across the origin, therefore, as an immediate consequence of (5.5) we get control over the first terms appearing in (4.14)

$$
e^{U_{j}-U_{i}}=\sqrt{\frac{\mathfrak{g}_{i}\left(\partial_{\varphi}, \partial_{\varphi}\right)}{\mathfrak{g}_{j}\left(\partial_{\varphi}, \partial_{\varphi}\right)}} \leq \sqrt{\frac{C_{i} \rho^{2}}{C_{j}^{-1} \rho^{2}}} \leq C \text { near }(0,0) \in \mathscr{A} \cap\left\langle\left\langle\mathscr{M}_{\text {ext }}\right\rangle\right\rangle .
$$

Since the $\chi_{i}$ 's and the $\psi_{i}$ 's are all bounded near the origin our goal gets reduced to showing that

$$
\rho^{-2} e^{U_{1}+U_{2}}\left(f_{1}-f_{2}\right)=O(1) \text { near }(0,0) \in \mathscr{A} \cap\left\langle\left\langle\mathscr{M}_{\text {ext }}\right\rangle\right\rangle,
$$

when $f_{1}=\chi_{1}, \psi_{1}, v_{1}, \chi_{1} \psi_{2}$ and $f_{2}=\chi_{2}, \psi_{2}, v_{2}, \chi_{2} \psi_{1}$, where by this we mean that if, for example, we set $f_{1}=\chi_{1}$ then $f_{2}=\chi_{2}$.

Let us start with $f_{1}=\chi_{1}, \psi_{1}, v_{1}$ and $f_{2}=\chi_{2}, \psi_{2}, v_{2}$. Each $f_{i}$ is invariant under the respective axial flow and constant along each connected component of $\mathscr{A}_{i} \cap$ $\left\langle\left\langle\mathscr{M}_{\text {ext }}\right\rangle\right\rangle$, so if we impose $f_{1}(0,0)=f_{2}(0,0)=f_{0}$, which is always achievable if the space-times $\left(\mathscr{M}_{i}, \mathfrak{g}_{i}\right)$ have the same set of masses, angular momenta and charges (see 
Section 6 and [36, Section 2.3]), we see that (5.3) holds and using (5.5) and (5.6) we get

$$
\begin{aligned}
\left|\rho^{-2} e^{U_{1}+U_{2}}\left(f_{1}-f_{2}\right)\right| & =\left|\frac{f_{1}-f_{2}}{\sqrt{\mathfrak{g}_{1}\left(\partial_{\varphi}, \partial_{\varphi}\right)} \sqrt{\mathfrak{g}_{2}\left(\partial_{\varphi}, \partial_{\varphi}\right)}}\right| \leq \frac{\left|f_{1}-f_{0}\right|+\left|f_{2}-f_{0}\right|}{\sqrt{\mathfrak{g}_{1}\left(\partial_{\varphi}, \partial_{\varphi}\right)} \sqrt{\mathfrak{g}_{2}\left(\partial_{\varphi}, \partial_{\varphi}\right)}} \\
& =\frac{\left|f_{1}-f_{0}\right|}{\mathfrak{g}_{1}\left(\partial_{\varphi}, \partial_{\varphi}\right)} \sqrt{\frac{\mathfrak{g}_{1}\left(\partial_{\varphi}, \partial_{\varphi}\right)}{\mathfrak{g}_{2}\left(\partial_{\varphi}, \partial_{\varphi}\right)}}+\frac{\left|f_{2}-f_{0}\right|}{\mathfrak{g}_{2}\left(\partial_{\varphi}, \partial_{\varphi}\right)} \sqrt{\frac{\mathfrak{g}_{2}\left(\partial_{\varphi}, \partial_{\varphi}\right)}{\mathfrak{g}_{1}\left(\partial_{\varphi}, \partial_{\varphi}\right)}} \\
& \leq \frac{C_{1} \hat{x}_{1}^{2}}{C_{2}^{-1} \hat{x}_{1}^{2}} C_{3}+\frac{C_{4} \hat{x}_{2}^{2}}{C_{5}^{-1} \hat{x}_{2}^{2}} C_{6} \\
& \leq C \text { near }(0,0) \in \mathscr{A} \cap\left\langle\left\langle\mathscr{M}_{\mathrm{ext}}\right\rangle\right\rangle .
\end{aligned}
$$

We take the chance to stress the fact that the previous argument does not apply to the fields $\chi_{1} \psi_{2}$ and $\chi_{2} \psi_{1}$ since these products involve functions originating from different space-times and therefore only make sense as functions of $(\rho, z)$ for which estimates like 5.3 are not available a priori $(7)$

To bypass this problem we write

$$
\chi_{1} \psi_{2}-\chi_{2} \psi_{1}=\left(\chi_{1}+\chi_{2}\right)\left(\psi_{2}-\psi_{1}\right)+\chi_{1} \psi_{1}-\chi_{2} \psi_{2} .
$$

Since $\chi_{1}+\chi_{2}$ is bounded, to control the first term we just need to take $f_{i}=\psi_{i}$ as before. Setting $f_{1}=\chi_{1} \psi_{1}$ and $f_{2}=\chi_{2} \psi_{2}$ we see that the previous argument still applies as these are also axially symmetric functions which are constant along the axis components. The desired result follows.

To finish the proof of boundedness of (4.14) near the singular set $\mathscr{A} \cap\left\langle\left\langle\mathscr{M}_{\text {ext }}\right\rangle\right\rangle$ we still have to analyze what happens near points where the axis meets the horizon. Choose such a point in each space-time and, without loss of generality, assume that these are 'north poles' which, as before, lie at the origin of the coordinate systems $\left(\hat{x}_{i}, \hat{z}_{i}\right)$, and satisfy $(\rho, z)=\Psi_{i}(0,0)=(0,0)$.

As already mentioned, a careful extension of the doubling of the orbits spaces validates (5.3) in a neighborhood of these 'north poles', but, on the other hand, the $\rho_{i}$ 's are now non-differentiable at such points and (5.6) no longer holds. Nonetheless, if we are able to control $e^{U_{i}-U_{j}}$ by other means, then the inequalities established in (5.8) extend to the case under consideration and boundedness of the distance near the axis follows. This problem, which is in fact the major difficulty that arises in the analysis of the boundary conditions of this axisymmetric harmonic maps, has been recently overcomed for the vacuum case [13, Section 6.5 .1$]$ by obtaining the following uniform estimate

$$
U=\ln \sqrt{z+\sqrt{z^{2}+\rho^{2}}}+O(1) \text { near }(0,0) \in \mathscr{A} \cap \mathscr{E}^{+},
$$

from which the desired consequence immediately follows. This result, which requires this component of the horizon to be non-degenerate, extends to the electro-vacuum case immediately.

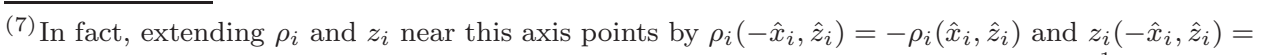
$z_{i}\left(\hat{x}_{i}, \hat{z}_{i}\right)$ shows that invariance under the axial flow implies that $f(\rho, z):=f \circ \Psi_{i}^{-1}(\rho, z)$ is an even function of $\rho$. Then, direct estimates in terms of $\rho$ analogous to (5.3) may be obtained for all the fields and the presented procedure including (5.8) may be bypassed. Unfortunately this is no longer possible near points where the axis meets the horizon as the $\rho_{i}$ are no longer differentiable.
} 
5.2. Spatial infinity. - In this section we want to show that

$$
\lim _{\sqrt{\rho^{2}+z^{2}} \rightarrow+\infty} d\left(\Phi_{1}, \Phi_{2}\right)=0,
$$

with $d$ implicitly defined by (4.14). For this we will assume stationarity and asymptotic flatness as given by the system of equations (2.12)-(2.15). It turns out that the estimates provided by asymptotic flatness, even in the way just defined, seem insufficient to control the relevant fields; even in an adapted frame provided by the results of Section 3 integration of the defining equations (4.1) yields divergent logarithmic terms. Fortunately, in the stationary and electro-vacuum setting, the asymptotic analysis of Beig and Simon [3,32] provides relevant improvements of the initial decay rates by means of the expansion (5.15).

Let $D$ and $\epsilon_{i j k}$ denote the covariant derivative and volume element of $\gamma$, the induced metric in $\mathscr{S}_{\text {ext }}$ as in Section 2. A well known consequence of the source free Einstein-Maxwell equations (2.1)-(2.3) and simple-connectedness of $\left\langle\left\langle\mathscr{M}_{\text {ext }}\right\rangle\right\rangle$ is the global existence of functions $\tau$ and $\sigma$ satisfying $[24,32,36]$

$$
D_{i} \tau=V^{2} \epsilon_{i}^{j k}\left(D_{j} A_{k}+\theta_{j} D_{k} A_{0}\right)
$$

and

$$
D_{i} \sigma=-V^{4} \epsilon_{i}{ }^{j k} D_{j} \theta_{k}+i\left(\bar{\Psi} \partial_{i} \Psi-\Psi \partial_{i} \bar{\Psi}\right),
$$

where $\Psi:=A_{0}+i \tau$. If we introduce the Ernst potential

$$
\mathscr{C}=V^{2}-\Psi \bar{\Psi}+i \sigma,
$$

and consider the complex valued fields $\zeta$ and $\vartheta$, implicitly defined by

$$
\mathscr{C}=\frac{1-\vartheta}{1+\vartheta} \quad, \quad \Psi=\frac{\zeta}{1+\vartheta},
$$

then [32, eq 3.11] provides the following expansion for the vector $\mathscr{E}^{A}:=(\vartheta, \zeta) \in \mathbb{C}^{2}$ in terms of an arbitrary asymptoticaly flat coordinate system

$$
\mathscr{E}^{A}=\frac{M^{A}}{r}+\frac{M_{k}^{A} x^{k}}{r^{3}}+O_{\infty}\left(\log r / r^{3}\right) .
$$

We note that the apparent discrepancy between the error term here with the one in the original paper comes from the fact that the result there is presented in adapted coordinates obtained from arbitrary asymptotically flat coordinates by a transformation of the form $x^{i} \mapsto x^{i}+O_{\infty}(\log r)$.

Using the identity $\frac{A}{B+C}=\frac{A}{B}-\frac{A C}{B(B+C)}$ we get

$$
\begin{aligned}
\mathscr{C} & =\frac{1-\vartheta}{1+\vartheta}=\frac{1+\vartheta-2 \vartheta}{1+\vartheta}=1-\frac{2 \vartheta}{1+\vartheta}=1-2\left(\vartheta-\frac{\vartheta^{2}}{1+\vartheta}\right) \\
& =1-2\left(\vartheta-\vartheta^{2}+\frac{\vartheta^{3}}{1+\vartheta}\right) .
\end{aligned}
$$

Inserting the $\vartheta$-component of (5.15) into the last expression yields

$$
\begin{aligned}
\mathscr{C} & =1-2\left(\frac{M^{\vartheta}}{r}+\frac{M_{k}^{\vartheta} x^{k}}{r^{3}}+O_{\infty}\left(\log r / r^{3}\right)\right)+2\left(\frac{M^{\vartheta}}{r}+\frac{M_{k}^{\vartheta} x^{k}}{r^{3}}+O_{\infty}\left(\log r / r^{3}\right)\right)^{2}+O_{\infty}\left(r^{-3}\right) \\
& =1-2 \frac{M^{\vartheta}}{r}-2 \frac{M_{k}^{\vartheta} x^{k}}{r^{3}}+2 \frac{\left(M^{\vartheta}\right)^{2}}{r^{2}}+O_{\infty}\left(r^{-4}\right)+O_{\infty}\left(\log r / r^{3}\right) .
\end{aligned}
$$

Noting that the topological restrictions imposed by asymptotic flatness imply that the imaginary part of $M^{\vartheta}$ vanishes, $\Im M^{\vartheta}=0\left[32\right.$, Section IV], we write $M^{\vartheta}=M$ 
and by setting $M_{k}^{\vartheta}=M_{k}+i S_{k}$ we get

$$
\mathscr{C}=1-2 \frac{M}{r}+2 \frac{M^{2}}{r^{2}}-2 \frac{M_{k} x^{k}}{r^{3}}-2 i \frac{S_{k} x^{k}}{r^{3}}+O_{\infty}\left(\log r / r^{3}\right) .
$$

Consequently

$$
\sigma=\Im \mathscr{C}=-2 \frac{S_{k} x^{k}}{r^{3}}+O_{\infty}\left(\log r / r^{3}\right)
$$

Similarly for $\Psi$ we get

$$
\Psi=\frac{\zeta}{1+\vartheta}=\zeta-\frac{\zeta \vartheta}{1+\vartheta}=\zeta-\zeta \vartheta+\frac{\zeta \vartheta^{2}}{1+\vartheta} .
$$

Inserting (5.15) into the last expression yields

$$
\begin{aligned}
\Psi & =\zeta(1-\vartheta)+O_{\infty}\left(r^{-3}\right) \\
& =\left(\frac{M^{\zeta}}{r}+\frac{M_{k}^{\zeta} x^{k}}{r^{3}}+O_{\infty}\left(\log r / r^{3}\right)\right)\left(1-\frac{M^{\vartheta}}{r}-\frac{M_{k}^{\vartheta} x^{k}}{r^{3}}+O_{\infty}\left(\log r / r^{3}\right)\right)+O_{\infty}\left(r^{-3}\right) \\
& =\frac{M^{\zeta}}{r}-\frac{M^{\zeta} M^{\vartheta}}{r^{2}}+\frac{M_{k}^{\zeta} x^{k}}{r^{3}}+O_{\infty}\left(\log r / r^{3}\right) .
\end{aligned}
$$

As before $\Im M^{v}=0$. So now, by setting $M^{\zeta}=\frac{Q}{2}$ and $M_{k}^{\zeta}=Q_{k}+i B_{k}$, we see that

$$
\begin{gathered}
A_{0}=\Re \Psi=\frac{Q}{2 r}-\frac{M Q}{2 r^{2}}+\frac{Q_{k} x^{k}}{r^{3}}+O_{\infty}\left(\log r / r^{3}\right), \\
\tau=\Im \Psi=\frac{B_{k} x^{k}}{r^{3}}+O_{\infty}\left(\log r / r^{3}\right) .
\end{gathered}
$$

We have $\bar{\Psi} \partial_{i} \Psi-\Psi \partial_{i} \bar{\Psi}=2 i\left(A_{0} \partial_{i} \tau-\tau \partial_{i} A_{0}\right)=O_{\infty}\left(r^{-4}\right)$ and using (2.14) and (5.17) we get

$$
\epsilon_{i}{ }^{j k} D_{j} \theta_{k}=-V^{-4}\left(D_{i} \sigma-i\left(\bar{\Psi} D_{i} \Psi-\Psi D_{i} \bar{\Psi}\right)\right)=D_{i}\left(2 \frac{S_{k} x^{k}}{r^{3}}+O_{\infty}\left(\log r / r^{3}\right)\right) .
$$

With the exception of the already noted $\log r$ discrepancy in the error term, this is [3, eq 4.1, pg 1010] and so we get

$$
\theta_{i}:=\frac{\mathfrak{g}_{i t}}{\mathfrak{g}_{t t}}=2 e_{i j k} \frac{S^{j} x^{k}}{r^{3}}+O_{k}\left(\log r / r^{3}\right),
$$

where $e_{[i j k]}=e_{i j k}$ with $e_{123}=1$.

5.2.1. The electromagnetic twist potential and the norm of the axial Killing vector. - Until now we have been working with a generic asymptotically flat coordinate system, but to estimate the electromagnetic twist potential $v$ via the Ernst equations (4.5) and the results of the previous section we will need to use adapted coordinates. So, let $\{t, \varphi, \rho, z\}$ be the Weyl coordinates as constructed in Section 3 and define the cylindrical type coordinates

$$
\left\{\begin{array}{l}
x=\rho \cos \varphi \\
y=\rho \sin \varphi
\end{array} .\right.
$$

A simple but noteworthy fact is that in this coordinate system we have

$$
K_{(1)}=\partial_{\varphi}=x \partial_{y}-y \partial_{x} .
$$

The estimates of the previous section will only be available to us in this coordinates if $\left\{t, x^{i}\right\}=\{t, x, y, z\}$ is an asymptotically flat coordinate system. This is in fact 
the case. To see it note that in the orbit space $\{t=\varphi=0\}$ the identity [13, eq 6.9] yields

$$
\begin{array}{cc}
\partial_{\rho}=\left(1+O_{k}\left(\hat{r}^{-1}\right)\right) \partial_{\hat{x}}+O_{\infty}\left(\hat{r}^{-1}\right) \partial_{\hat{z}} & (\varphi=0), \\
\partial_{z}=O_{\infty}\left(\hat{r}^{-1}\right) \partial_{\hat{x}}+\left(1+O_{\infty}\left(\hat{r}^{-1}\right)\right) \partial_{\hat{z}} & (\varphi=0) .
\end{array}
$$

Recall that $\{\hat{x}, \hat{z}\}$ are asymptotically flat isothermal coordinates (for the orbit space metric). Direct computations yield $\left.\mathfrak{g}_{x x}\right|_{\varphi=0}=1+O_{\infty}\left(r^{-1}\right)$, a similar expression for $\mathfrak{g}_{y y}$ and, using $\mathfrak{g}_{\rho \varphi} \equiv 0$, also $\left.\mathfrak{g}_{x y}\right|_{\varphi=0}=0$. The defining decay rates are now obtained by flowing the previous estimates along the integral lines of the axial Killing vector. We illustrate this with an explicit calculation:

$$
\begin{aligned}
\left.\mathfrak{g}_{x y}\right|_{\varphi=-\varphi_{0}} & =\left.\mathfrak{g}\left(\left(\phi_{\varphi_{0}}\right)_{*} \partial_{x},\left(\phi_{\varphi_{0}}\right)_{*} \partial_{y}\right)\right|_{\varphi=0} \\
& =\left.\mathfrak{g}\left(\cos \varphi_{0} \partial x+\sin \varphi_{0} \partial y,-\sin \varphi_{0} \partial x+\cos \varphi_{0} \partial y\right)\right|_{\varphi=0} \\
& =-\sin \varphi_{0} \cos \varphi_{0} \underbrace{\left.\mathfrak{g}_{x x}\right|_{\varphi=0}}_{=1+O_{\infty}\left(r^{-1}\right)}\left(\cos ^{2} \varphi_{0}-\sin ^{2} \varphi_{0}\right) \underbrace{\left.\mathfrak{g}_{x y}\right|_{\varphi=0}}_{=0}+\sin \varphi_{0} \cos \varphi_{0} \underbrace{\left.\mathfrak{g}_{y y}\right|_{\varphi=0}}_{=1+O_{\infty}\left(r^{-1}\right)} \\
& =O_{\infty}\left(r^{-1}\right) .
\end{aligned}
$$

So we have constructed asymptotically flat coordinates for which the following uniform estimate holds

$$
\left.\mathfrak{g}_{\varphi \varphi}\right|_{\varphi=0}=\left.\rho^{2} \mathfrak{g}_{y y}\right|_{\varphi=0}=\rho^{2}\left(1+O_{\infty}\left(r^{-1}\right)\right) .
$$

As a nice consequence we get

$$
e^{-2 U}:=\frac{\mathfrak{g}_{\varphi \varphi}}{\rho^{2}}=1+O_{\infty}\left(r^{-1}\right)
$$

from which we see that

$$
e^{U_{i} \pm U_{j}}:=\left(1+O_{\infty}\left(r^{-1}\right)\right)\left(1+O_{\infty}\left(r^{-1}\right)\right)^{ \pm 1}=1+O_{\infty}\left(r^{-1}\right) \rightarrow_{r \rightarrow+\infty} 1,
$$

and our goal (5.10) gets reduced to showing that

$$
\begin{aligned}
\lim _{\sqrt{\rho^{2}+z^{2}} \rightarrow+\infty} \frac{\left(\psi_{1}-\psi_{2}\right)^{2}}{\rho^{2}} & =\lim _{\sqrt{\rho^{2}+z^{2}} \rightarrow+\infty} \frac{\left(\chi_{1}-\chi_{2}\right)^{2}}{\rho^{2}} \\
& =\lim _{\sqrt{\rho^{2}+z^{2}} \rightarrow+\infty} \frac{v_{1}-v_{2}}{\rho^{2}} \\
& =\lim _{\sqrt{\rho^{2}+z^{2}} \rightarrow+\infty} \frac{\chi_{1} \psi_{2}-\chi_{2} \psi_{1}}{\rho^{2}}=0 .
\end{aligned}
$$

It follows from (5.21) and (3.17) that

$$
\mathfrak{g}_{z t} \equiv 0 \Rightarrow \theta_{z} \equiv 0 \Rightarrow S_{x}=S_{y}=0 .
$$

So we set $J:=-S_{z}$ and by using (5.21) with (2.14) we get

$$
\left.\mathfrak{g}_{y t}\right|_{\varphi=0}=2 J \frac{\rho}{r^{3}}+O_{\infty}\left(\log r / r^{3}\right),
$$

from which

$$
\left.\mathfrak{g}_{\varphi t}\right|_{\varphi=0}=\left.\mathfrak{g}\left(\rho \partial_{y}, \partial_{t}\right)\right|_{\varphi=0}=2 J \frac{\rho^{2}}{r^{3}}+\rho O_{\infty}\left(\log r / r^{3}\right),
$$

and therefore

$$
\left.\frac{\mathfrak{g}_{\varphi t}}{\mathfrak{g}_{\varphi \varphi}}\right|_{\varphi=0}=\frac{2 J}{r^{3}}+\frac{1}{\rho} O_{\infty}\left(\log r / r^{3}\right) .
$$


The Ernst equations (4.5) together with the estimates (5.33), (5.35), (5.47) and (5.48), that will be established in the next section, provide

$$
\left\{\begin{array}{l}
\partial_{z} v=-6 J \rho^{4} / r^{5}+\rho O_{\infty}\left(\log r / r^{3}\right) \\
\partial_{\rho} v=6 J \rho^{3} z / r^{5}+\rho^{2} O_{\infty}\left(\log r / r^{4}\right)
\end{array} .\right.
$$

Integrating this system by using the polar coordinates $\rho=r \cos \theta, z=r \sin \theta$, while imposing the standard condition

$$
v(0, z) \equiv 0, \text { for } z \gg 0,
$$

yields

$$
v=4 J-\frac{J}{2} \frac{z}{r}\left(\frac{3 \rho^{2}-z^{2}}{r^{2}}+9\right)+\rho O_{\infty}\left(\log r / r^{2}\right) .
$$

We note the following relevant relation with the total angular momentum as given by the Komar integral formula

$$
\begin{aligned}
\lim _{R \rightarrow+\infty} \frac{1}{16 \pi} \int_{\{r=R\}} * d K_{(1)}^{b} & =\lim _{R \rightarrow+\infty}-\frac{1}{16 \pi} 2 \pi \int_{\{r=R\} \cap\{\varphi=0\}} i_{K_{(1)}} * d K_{(1)}^{b} \\
& =-\frac{1}{8} \lim _{R \rightarrow+\infty} \int_{\{r=R\} \cap\{\varphi=0\}} *\left(d K_{(1)}^{b} \wedge K_{(1)}^{b}\right) \\
& =-\frac{1}{8} \lim _{R \rightarrow+\infty} \int_{\{r=R\} \cap\{\varphi=0\}}(d v+2 \underbrace{(\chi d \psi-\psi d \chi)}_{=O\left(r^{-2}\right)}) \\
& =-\frac{1}{8} \lim _{R \rightarrow+\infty}(v(0, R)-v(0,-R)) \\
& =-\frac{1}{8}(0-8 J) \\
& =J .
\end{aligned}
$$

We are now able to establish the electromagnetic twist potential part of (5.29). For two twist potentials satisfying (5.34) we have

$$
\lim _{\sqrt{\rho^{2}+z^{2}} \rightarrow+\infty, \rho \nrightarrow 0} \frac{v_{1}-v_{2}}{\rho^{2}}=0 .
$$

To take care of the asymptotic behavior of $v$ near the axis we Taylor expand on $\rho$ around a point $(0, z)$, away from the poles, to get

$$
v(\rho, z)=v(0, z)+\partial_{\rho} v(c(\rho), z) \rho \quad,|c(\rho)| \leq \rho .
$$

Then, using (5.33) to obtain

$$
\partial_{\rho} v=\rho^{2} O_{\infty}\left(r^{-3}\right),
$$

we conclude that for $|z| \gg 0$ and $\rho \leq|z|$

$$
v=v(0, z)+\rho^{2} O\left(r^{-3}\right) .
$$

Finally, for two twist potentials that agree along the axis for both large positive and negative $z$ we have, in the region $\rho \leq|z|$,

$$
\rho^{-2}\left(v_{1}-v_{2}\right)=O\left(r^{-3}\right),
$$

and the desired result follows. 
5.2.2. The electromagnetic potentials. - Asymptotic flatness (2.7) together with (5.18) and (5.19) yield the desired improvement of the initial decay rates

$$
\partial_{[i} A_{j]}=O_{\infty}\left(r^{-3}\right) .
$$

Now in the $\left\{x^{\mu}\right\}=\{t, x, y, z\}$ coordinates of the previous section we have

$$
\begin{aligned}
d \chi & :=i_{K_{(1)}} F \\
& =F_{\mu \nu} d x^{\mu} d x^{\nu}\left(K_{(1)}, \cdot\right) \\
& =F_{\mu \nu}\left(d x^{\mu}\left(K_{(1)}\right) d x^{\nu}-d x^{\nu}\left(K_{(1)}\right) d x^{\mu}\right)=2 F_{\mu \nu} d x^{\mu}\left(K_{(1)}\right) d x^{\nu} \\
& =2 F_{\mu \nu} d x^{\mu}\left(x \partial_{y}-y \partial_{x}\right) d x^{\nu}=2 F_{\mu \nu}\left(x \delta_{y}^{\mu}-y \delta_{x}^{\mu}\right) d x^{\nu} \\
& =2\left(x F_{y \nu}-y F_{x \nu}\right) d x^{\nu}=4\left(x \partial_{[y} A_{\nu]}-y \partial_{[y} A_{\nu]}\right) d x^{\nu} .
\end{aligned}
$$

With (5.41) we see that, in the orbit space $\{\varphi=0\}$ (where $y=0, x=\rho$ and $\partial_{x}=\partial_{\rho}$ ), we have

$$
\left\{\begin{array}{l}
\left.\partial_{\rho} \chi\right|_{\varphi=0}=4 \rho \partial_{[y} A_{\rho]}=\rho O_{\infty}\left(r^{-3}\right) \\
\left.\partial_{z} \chi\right|_{\varphi=0}=4 \rho \partial_{[y} A_{z]}=\rho O_{\infty}\left(r^{-3}\right)
\end{array} .\right.
$$

Imposing the boundary condition

$$
\chi(0, z) \equiv 0, \text { for } z \gg 0,
$$

integration yields

$$
\left.\chi\right|_{\varphi=0}=\rho O_{\infty}\left(r^{-2}\right) .
$$

Arguing as in the end of section 5.2.1 the equation in (5.29) corresponding to the potentials $\chi_{i}$ follows.

To obtain a coordinate expression for $d \psi$ it will be helpful to rearrange our preferred coordinate system and consider $\left\{x^{\mu}\right\}=\{t, y, x, z\}$, then

$$
\begin{aligned}
d \psi & :=i_{K_{(1)}} * F \\
& =\frac{1}{2} F^{\mu \nu} \epsilon_{\mu \nu \lambda \sigma} d x^{\lambda} d x^{\sigma}\left(K_{(1)}, \cdot\right)=F^{\mu \nu} \epsilon_{\mu \nu \lambda \sigma} d x^{\lambda}\left(x \partial_{y}-y \partial_{x}\right) d x^{\sigma} \\
& =F^{\mu \nu} \epsilon_{\mu \nu \lambda \sigma}\left(x \delta_{y}^{\lambda}-y \delta_{x}^{\lambda}\right) d x^{\sigma}=F^{\mu \nu}\left(x \epsilon_{\mu \nu y \sigma}-y \epsilon_{\mu \nu x \sigma}\right) d x^{\sigma} .
\end{aligned}
$$

Now, in the orbit space and away from the axis, we have (compare with (3.17)

$$
\left.\mathfrak{g}_{\mu \nu}\right|_{\varphi=0}=\left(\begin{array}{cccc}
\mathfrak{g}_{t t} & \rho^{-1} \mathfrak{g}_{t \varphi} & 0 & 0 \\
\rho^{-1} \mathfrak{g}_{t \varphi} & \rho^{-2} \mathfrak{g}_{\varphi \varphi} & 0 & 0 \\
0 & 0 & e^{2 u} & 0 \\
0 & 0 & 0 & e^{2 u}
\end{array}\right)
$$

therefore

$$
\operatorname{det}\left(\left.\mathfrak{g}_{\mu \nu}\right|_{\varphi=0}\right)=\left(\mathfrak{g}_{t t} \frac{\mathfrak{g}_{\varphi \varphi}}{\rho^{2}}-\frac{\mathfrak{g}_{t \varphi}^{2}}{\rho^{2}}\right) e^{4 u}=\frac{1}{\rho^{2}}\left(-\rho^{2}\right) e^{4 u}=-e^{4 u}
$$

so

$$
\begin{aligned}
\left.\partial_{\rho} \psi\right|_{\varphi=0} & =\rho \epsilon_{\mu \nu y x} F^{\mu \nu}=\rho\left(\epsilon_{t z y x} F^{t z}+\epsilon_{z t y x} F^{z t}\right)=2 \rho \epsilon_{t z y x} F^{t z} \\
& =2 \rho \sqrt{\left|\operatorname{det}\left(\mathfrak{g}_{\mu \nu}\right)\right|} F^{t z}=2 \rho e^{2 u} \mathfrak{g}^{\mu t} \mathfrak{g}^{\nu z} F_{\mu \nu} \\
& =2 \rho e^{2 u} \mathfrak{g}^{\mu t} \mathfrak{g}^{z z} F_{\mu z}=2 \rho e^{2 u} e^{-2 u}\left(\mathfrak{g}^{t t} F_{t z}+\mathfrak{g}^{y t} F_{y z}\right) \\
& =2 \rho\left\{-\frac{\mathfrak{g}_{\varphi \varphi}}{\rho^{2}}\left(\partial_{t} A_{z}-\partial_{z} A_{t}\right)+2 \frac{\mathfrak{g}_{t \varphi}}{\rho} \partial_{[y} A_{z]}\right\} \\
& =2 \frac{\mathfrak{g}_{\varphi \varphi}}{\rho} \partial_{z} A_{t}+4 \mathfrak{g}_{t \varphi} \partial_{[y} A_{z]}=2 \rho\left(1+O_{\infty}\left(r^{-1}\right)\right) \partial_{z} A_{0}+\rho O_{\infty}\left(r^{-5}\right),
\end{aligned}
$$


where in the last equality we used (5.26), (5.31) and (5.41); also

$$
\begin{aligned}
\left.\partial_{z} \psi\right|_{\varphi=0} & =\rho \epsilon_{\mu \nu y z} F^{\mu \nu}=2 \rho \epsilon_{t x y z} F^{t x} \\
& =-2 \rho \sqrt{\left|\operatorname{det}\left(\mathfrak{g}_{\mu \nu}\right)\right|} F^{t x}=-2 \rho e^{2 u} \mathfrak{g}^{\mu t} \mathfrak{g}^{\nu x} F_{\mu \nu} \\
& =-2 \rho e^{2 u} \mathfrak{g}^{\mu t} \mathfrak{g}^{x x} F_{\mu x}=-2 \rho\left(\mathfrak{g}^{t t} F_{t x}+\mathfrak{g}^{y t} F_{y x}\right) \\
& =-2 \rho\left\{-\frac{\mathfrak{g}_{\varphi \varphi}}{\rho^{2}}\left(\partial_{t} A_{x}-\partial_{x} A_{t}\right)+2 \frac{\mathfrak{g}_{t \varphi}}{\rho} \partial_{[y} A_{x]}\right\} \\
& =-2 \rho\left(1+O_{\infty}\left(r^{-1}\right)\right) \partial_{\rho} A_{0}+\rho O_{\infty}\left(r^{-5}\right) .
\end{aligned}
$$

From (5.18) we get

$$
\left\{\begin{array}{l}
\left.\partial_{\rho} \psi\right|_{\varphi=0}=-Q \frac{\rho z}{r^{3}}+\rho O_{\infty}\left(r^{-3}\right) \\
\left.\partial_{z} \psi\right|_{\varphi=0}=Q \frac{\rho^{2}}{r^{3}}+\rho O_{\infty}\left(r^{-3}\right)
\end{array} .\right.
$$

Integrating as before while using a standard boundary condition provides

$$
\psi=Q\left(-1+\frac{z}{r}\right)+\rho O_{\infty}\left(r^{-2}\right) .
$$

We note the following relevant and expected relation with the total electric charge given by the Komar integral

$$
\lim _{R \rightarrow+\infty}-\frac{1}{4 \pi} \int_{\{r=R\}} * F=Q .
$$

It should be now clear that (5.29) follows.

The results of this last two sections establish one of the significant missing elements of all previous uniqueness claims for the Kerr-Newman metric:

Proposition 5.1. - Let $\Psi_{i}=\left(U_{i}, v_{i}, \chi_{i}, \psi_{i}\right), i=1,2$, be the Ernst potentials

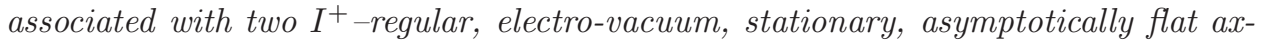
isymmetric metrics with non-degenerate event horizons. If $v_{1}=v_{2}, \psi_{1}=\psi_{2}$ and $\chi_{1}=\chi_{2}$ on the rotation axis, then the hyperbolic-space distance between $\Psi_{1}$ and $\Psi_{2}$ is bounded, going to zero as $r$ tends to infinity in the asymptotic region.

\section{Weinstein Solutions: existence and uniqueness}

In this section we construct axisymmetric Ernst maps

$$
\Phi=(U, v, \chi, \psi): \mathbb{R}^{3} \backslash \mathscr{A} \rightarrow \mathbb{H}_{\mathbb{C}}^{2},
$$

which are "close" to some reference maps, not necessarily harmonic, satisfying conditions modeled on the local behavior of the Kerr-Newman solutions. First recall the definitions of mass, angular momentum and electric charge of the $k$-th black hole as given by the Komar integrals

$$
\begin{aligned}
m_{k} & :=-\frac{1}{8 \pi} \int_{S_{k}} * d K_{(0)}^{b}, \\
J_{k} & :=\frac{1}{16 \pi} \int_{S_{k}} * d K_{(1)}^{b}, \\
q_{k} & :=-\frac{1}{4 \pi} \int_{S_{k}} * F .
\end{aligned}
$$

for some 2 -sphere $S_{k}$ whose interior intersects the event horizon exactly at its $k$-th component. 
We are now able to characterize the reference maps $\tilde{\Phi}=(\tilde{U}, \tilde{v}, \tilde{\chi}, \tilde{\psi})$ :

1. The components $\tilde{f}=\tilde{v}, \tilde{\chi}$ and $\tilde{\psi}$ are locally bounded, constant along each connected component of $\mathscr{A} \backslash \mathscr{E}^{+}=\cup_{k=0}^{N} \mathscr{A}_{k}$ and we write $\left.\tilde{f}\right|_{\mathscr{A}_{k}} \equiv \tilde{f}_{k}$. These functions are normalized to satisfy $\tilde{f}_{N}=0$.

2. There exist $N_{\mathrm{dh}} \geq 0$ degenerate event horizons, which are represented by punctures $\left(\varphi=0, \rho=0, z=b_{i}\right)$, together with a mass parameter $m_{i}>0$. In a neighborhood of such puncture, containing only this component of the horizon, the map $\tilde{\Phi}$ corresponds to the harmonic map of the (extreme) KerrNewman solution parameterized by

$$
\left(m_{i}, q_{i}\right)=\left(m_{i}, \frac{\tilde{\psi}_{i+1}-\tilde{\psi}_{i}}{2}\right) .
$$

3. There exist $N_{\text {ndh }} \geq 0$ non-degenerate horizons, which are represented by bounded open intervals $\left(c_{i}^{-}, c_{i}^{+}\right)=I_{i} \subset \mathscr{A}$, with none of the previous $b_{j}$ 's belonging to the union of the closures of the $I_{i}$. In a neighborhood of such interval, containing only this component of the horizon, the map $\tilde{\Phi}$ corresponds to the harmonic map of the Kerr-Newman solution parameterized by

$$
\left(\mu_{j}, \lambda_{j}, q_{j}\right)=\left(2 \int_{I_{j}} d z, \tilde{v}_{i+1}-\tilde{v}_{i}, \frac{\tilde{\psi}_{i+1}-\tilde{\psi}_{i}}{2}\right) .
$$

To retrieve the usual parametrization using mass, angular mommentum and charge one uses the known explicit formulas for Kerr-Newman (e.g., equations 2.31. of [36]) together with the following relations [36, section 2.3.]

$$
\begin{gathered}
J_{j}=\frac{\lambda_{j}+l_{j}}{4}, \\
m_{j}=\mu_{j}+2 w_{j} J_{j},
\end{gathered}
$$

where the auxiliary parameters are defined by $\lambda_{j}:=\int_{I_{j}} d v, l_{j}=\int_{I_{j}} \chi d \psi-\psi d \chi$, and $\left.w\right|_{I_{j}} \equiv w_{j}$, with $w$ defined by (3.17).

4. In a neighborhood of infinity the functions $\tilde{U}, \tilde{v}, \tilde{\chi}$ and $\tilde{\psi}$ coincide with the components of the harmonic map associated with the Kerr-Newman solution with mass $M:=\sum_{k} m_{k}$ angular momentum $J:=\sum_{k} J_{k}=v_{0} / 8$ and electric charge $Q:=\sum_{k} q_{k}=-\psi_{0} / 2$, where the sums are taken over all the components of the event horizon.

5. The functions $\tilde{U}, \tilde{v}, \tilde{\chi}$ and $\tilde{\psi}$ are smooth across $\mathscr{A} \backslash\left(\cup_{i}\left\{b_{i}\right\} \cup_{j} I_{j}\right)$.

A collection $\left\{b_{i}, m_{i}\right\}_{i=1}^{N_{\mathrm{dh}}},\left\{I_{j}, v\left(c_{j}^{-}\right), v\left(c_{j}^{+}\right)\right\}_{j=1}^{N_{\mathrm{dh}}}$, and $\left\{\psi_{k}\right\}_{k=0}^{N-1}$ will be called "electrovacuum axis data".

A map $\tilde{\Phi}$ satisfying condition 1.-5. above defines singular Dirichlet data [37, Definition 2] (compare [36, Section 2.4.]) with a target manifold with constant negative sectional curvature. We then have the following version of [37, Theorem 2] (compare [12, Appendix C] where the uniqueness claim is clarified, and [36] for a similar result stated purely in terms of axis data):

Theorem 6.1. - For any set of electro-vacuum axis data there exists a unique harmonic map $\Phi: \mathbb{R}^{3} \backslash \mathscr{A} \rightarrow \mathbb{H}_{\mathbb{C}}^{2}$ whose distance, as given by (4.14), from an axisymmetric map $\tilde{\Phi}: \mathbb{R}^{3} \backslash \mathscr{A} \rightarrow \mathbb{H}_{\mathbb{C}}^{2}$, not necessarily harmonic but with the properties 1.-5. above, satisfies:

$$
d(\Phi, \tilde{\Phi}) \in L^{\infty}\left(\mathbb{R}^{3} \backslash \mathscr{A}\right)
$$


and

$$
d(\Phi, \tilde{\Phi}) \rightarrow 0 \text { as } r \rightarrow+\infty
$$

From an harmonic map $\Phi: \mathbb{R}^{3} \backslash \mathscr{A} \rightarrow \mathbb{H}_{\mathbb{C}}^{2}$ one can construct a stationary and axisymmetric solution of the source free Einstein-Maxwell field equations [36, Section 4.1.]. Such (not necessarily $I^{+}-$regular) space-times, arising from the harmonic maps of the previous theorem will be referred to as Weinstein solutions.

\section{Proof of Theorem 1.3}

If $\mathscr{E}^{+}$is empty we obtain Minkowski by an aplication of [7, Theorem 2.7]. Otherwise the proof splits into two cases, according to whether or not $K$ is tangent to the generators of $\mathscr{E}^{+}$.

\section{Rotating horizons:}

Suppose, first, that the Killing vector is not tangent to the generators of some connected component $\mathscr{E}_{0}^{+}$. Proposition 1.9 of [7] allows us to generalize [13, Proposition 4.10] to electro-vacuum and then Theorem 4.11 together with the Remark 4.12 of [13] show that the event horizon is analytic if the metric is; also, by (5.41) and Einstein's equations, $G_{\mu \nu}=2 T_{\mu \nu}=O\left(r^{-5}\right)$. So the Rigidity Theorem, as presented in [8, Theorem 5.1], applies and establishes the existence of a $\mathbb{R} \times \mathrm{U}(1)$ subgroup of the isometry group of $(\mathscr{M}, \mathfrak{g})$. The analysis of Section 3 , leading to the global representation (3.17) of the metric, is now available. As stressed throughout this paper, in this gauge, the field equations (2.1)-(2.3) reduce to a harmonic map $\Phi$ (4.3). The analysis of the asymptotic behavior of such map, whose results are compiled in Proposition 5.1 shows that $\Phi$ lies a finite distance from one of the harmonic maps associated to the Weinstein solutions of Theorem 6.1 and the uniqueness part of such theorem allows us to conclude; note that in the connected and non-degenerate setting the Weinstein solutions correspond to the non-extreme Kerr-Newman metrics.

\section{Non-rotating case:}

Now let us consider the case when the stationary Killing vector $K_{(0)}$ is tangent to the generators of every component of $\mathscr{E}^{+}$. Following the procedure in [13, Section 7.2], based on [30], we extend $\left\langle\left\langle\mathscr{M}_{\text {ext }}\right\rangle\right\rangle$ to a space-time where each connected component of the event horizon is contained in a bifurcate horizon. Then, by [15] there exists an asymptotically flat Cauchy hypersurface for the domain of outer communications, with boundary on the union of the bifurcate spheres, which is maximal. We are now able to conclude from Theorem 3.4 of [33] that $\left\langle\left\langle\mathscr{M}_{\text {ext }}\right\rangle\right\rangle$ is static. By taking in account the corrections presented in the proof of Theorem 1.4 of [13] we can invoke, after relying on analyticity once more, the non-degenerate part of the conclusion of Theorem 1.3 of [9], yielding non-extreme Reissner-Nordström as the only non-rotating solution satisfying the remaining conditions of the desired result.

\section{Concluding remarks}

To obtain a satisfactory classification in four dimensions, the following issues remain to be addressed: 
1. Analyticity. The previous versions of the uniqueness theorem required analyticity of both the metric and the horizon. As shown in the proof of Theorem 1.3. the latter follows from the former. This is a worthwhile improvement, as even $C^{1}$-differentiability of the horizon is not clear a priori. But the hypothesis of analyticity of the metric remains to be removed. In this context one should keep in mind the Curzon solution, where analyticity of the metric fails precisely at the horizon.

We further note that a new approach to Hawking's rigidity without analyticity $[1,2]$ as yield significant breakthroughs in the vacuum case. According to A.D. Ionescu (private communication) the generalization of the results to electro-vacuum should follow by similar techniques. However, some problems still need to be settled, even for vacuum: the local claim requires a nonexpanding horizon which we expect to be a consequence of $I^{+}$-regularity and the results and techniques of $[11,13]$, but such claim requires checking; also, as it stands, the global result is restricted to near Kerr geometries.

The hypothesis of analyticity is particularly annoying in the static context, being needed there only to exclude non-embedded Killing prehorizons [13, Section 5]. The nature of that problem seems to be rather different from Hawking's rigidity, with presumably a simpler solution, yet to be found.

2. Degeneracy. The classification of black holes with degenerate components of the event horizon requires further investigations. We believe that the results here go a long way to obtain a classification, in terms of Weinstein solutions, of stationary, axisymmetric, rotating configurations allowing both degenerate and non-degenerate components of the horizon: the foundations are settled but we are still missing an equivalent of Proposition 5.1. Recall that in the static case a complete classification in terms of the MajumdarPapapetrou and the Reissner-Norsdtröm families, with neither degeneracy or connectedness assumptions is already available by the work in [17] and references therein. In fact more is known in the degenerate class, since it was established in [14] that appropriately regular, $I^{+}$-regular in particular, IsraelWilson-Perjés Black holes belong to the Majumdar-Papapetrou family.

It has been announced [23] that the question of uniqueness of degenerate black holes (with connected event horizon) has been settled. Unfortunately, that reference does not contain any new results, as compared to what had already been published in [13], or is contained in this work, and so, it is our belief that this problem remains open. Indeed, the existence of global Weyl coordinates with controlled behavior at the singular set is assumed. In the non-degenerate case this issue was first settled for vacuum in [13], but the degenerate case appears to present serious technical difficulties, and requires further study.

3. Multi Component Solutions. In agreement with the statement of Conjecture 1.1, one believes that all solutions with non-connected $\mathscr{E}^{+}$are in the Majumdar-Papapetrou family. From what was said in the previous item, we see that it remains to show that non-static Weinstein solutions with nonconnected horizons are singular; besides the already quoted result dealing with the Israel-Wilson-Perjés family, this has been established for slowly rotating black holes in vacuum by a regularity analysis of the relevant harmonic maps $[25,34]$ and recent and promising results seem to have settled the problem for two-body configurations, also in vacuum [28]. 
Acknowledgements: We are grateful to Piotr Chruściel and José Natário for numerous comments on a previous version of the paper and many useful discussions.

\section{References}

[1] S. Alexakis , A. Ionescu \& S. Klainerman - "Hawking's local rigidity theorem without analyticity", (2009), arXiv:0902.1173v1 [gr-qc].

[2] S. Alexakis, A. Ionescu \& S. Klainerman - "Uniqueness of smooth stationary black holes in vacuum: small perturbations of the Kerr spaces ", (2009), arXiv:0904.0982 1 [gr-qc].

[3] R. Beig \& W. Simon - "The stationary gravitational field near spatial infinity. Gen. Relativity Gravitation 12 (1980), , no. 12, 1003-1013.

[4] B. CARTER - "Killing horizons and orthogonally transitive groups in space-time", Jour. Math. Phys. 10 (1969), p. 70-81.

[5] _ "Black hole equilibrium states", Black Holes (C. de Witt \& B. de Witt, eds.), Gordon \& Breach, New York, London, Paris, 1973, Proceedings of the Les Houches Summer School.

[6] _ "Has the black hole equilibrium problem been solved?", The Eighth Marcel Grossmann Meeting, Part A, B (Jerusalem, 1997), 136-155, World Sci. Publ., River Edge, NJ, (1999), arXiv:gr-qc/9712038

[7] P. Chrusciel - "No hair' theorems - folklore, conjectures, results.", Contemp. Math. Differential geometry and mathematical physics (Vancouver, BC, 1993), 23-49, Contemp. Math., 170, Amer. Math. Soc., Providence, RI, (1994), arXiv:gr-qc/9402032 [gr-qc].

[8] _ "Uniqueness of black holes revisited", Helv. Phys. Acta 69 (1996), p. 529552, Proceedings of Journés Relativistes 1996, Ascona, May 1996, N. Straumann,Ph. Jetzer and G. Lavrelashvili (Eds.), arXiv:gr-qc/9610010.

[9] - "Towards a classification of static electrovacuum spacetimes containing an asymptotically flat spacelike hypersurface with compact interior", Class. Quantum Grav. 16 (1999), p. 689-704.

[10] "Mass and angular-momentum inequalities for axi-symmetric initial data sets. I. Positivity of mass", Annals Phys. 323 (2008), p. 2566-2590, doi:10.1016/j.aop.2007.12.010, arXiv:0710.3680 [gr-qc].

[11] P. Chruściel, E. Delay, G. Galloway \& R. Howard - "Regularity of horizons and the area theorem", Annales Henri Poincaré 2 (2001), p. 109-178, arXiv:gr-qc/0001003.

[12] P. Chruściel, Y. Li \& G. Weinstein - "Mass and angular-momentum inequalities for axi-symmetric initial data sets. II. Angular momentum", Annals Phys. 323 (2008), p. 2591-2613, doi:10.1016/j.aop.2007.12.011, arXiv:0712.4064v2 [gr-qc].

[13] P. Chruściel \& J.L. Costa - "On uniqueness of stationary vacuum black holes." Géométrie Différentielle, Physique Mathématique, Mathématique et Société, Volume en l'honneur de Jean Pierre Bourguignon (O. Hjazi, éditeur), Astrisque 321, 2008, p. 195-265. http://arxiv.org/abs/0806.0016

[14] P. Chruściel, H. Reall \& K. Tod - "On Israel-Wilson-Perjs black holes.", Class. Quantum Grav. 23 (2006), p. 2519-2540, arXiv:gr-qc/0512116v1.

[15] P. Chruściel \& R. WALd - "Maximal hypersurfaces in stationary asymptotically flat space-times", Commun. Math. Phys. 163 (1994), p. 561-604, arXiv:gr-qc/9304009

[16] _ "On the topology of stationary black holes", Class. Quantum Grav. 11 (1994), p. L147-152, arXiv:gr-qc/9410004.

[17] P. Chruściel \& K. TOD - "The classification of static electro-vacuum space-times containing an asymptotically flat spacelike hypersurface with compact interior.", Comm. Math. Phys. 271 (2007), no. 3, 577-589, arXiv:gr-qc/0512043v2.

[18] S. DAIN - "Proof of the angular momentum-mass inequality for axisymmetric black holes", Jour. Diff. Geom. 79 (2006), p. 33-67, arXiv:gr-qc/0606105 
[19] G. Galloway - "On the topology of the domain of outer communication", Class. Quantum Grav. 12 (1995), p. L99-L101.

[20] _ "A "finite infinity" version of the FSW topological censorship", Class. Quantum Grav. 13 (1996), p. 1471-1478.

[21] S. Hawking - "Black holes in general relativity", Commun. Math. Phys. 25 (1972), p. $152-166$.

[22] M. Heusler - "Black hole uniqueness theorems", Cambridge University Press, Cambridge, 1996.

[23] A.J. Amsel , G.T. Horowitz , D. Marolf \& M.M. Roberts - "Uniqueness of Extremal Kerr and Kerr-Newman Black Holes", (2009), arXiv:0906.2367 [gr-qc].

[24] W. Israel \& G. Wilson- "A class of stationary electromagnetic vacuum fields", Jour. Math. Phys. 13 (1972), p. 865-867.

[25] Y. Li \& G. TIAN - "Regularity of harmonic maps with prescribed singularities", Commun. Math. Phys. 149 (1992), p. 1-30.

[26] P. Mazur - "Proof of uniqueness of the Kerr-Newman black hole solution", Jour. Phys. A: Math. Gen. 15 (1982), p. 3173-3180.

[27] _ "Black Hole uniqueness Theorems", General relativity and gravitation (Stockholm, 1986), 130-157, Cambridge Univ. Press, Cambridge, (1987), arXiv:hep-th/0101012

[28] G. Neugebauer \& J. Hennig - "Non-existence of stationary two-black-hole configurations", (2009), arXiv:0905.4179v3.

[29] P. OrLIK - "Seifert manifolds", Springer-Verlag, Berlin, 1972, Lecture Notes in Mathematics, Vol. 291.

[30] I. RÁcz \& R. WALD - "Global extensions of space-times describing asymptotic final states of black holes", Class. Quantum Grav. 13 (1996), p. 539-552, arXiv:gr-qc/9507055

[31] F. RAYMOND - "Classification of the actions of the circle on 3-manifolds", Trans. Amer. Math. Soc. 131 (1968), p. 51-78.

[32] W. Simon - "The Multipole expansion of stationary Einstein-Maxwell fields " J. Math. Phys. 25 (1984), no. 4, 1035-1038.

[33] D. Sudarsky \& R. WALD - "Extrema of mass, stationarity and staticity, and solutions to the Einstein-Yang-Mills equations", Phys. Rev. D46 (1993), p. 1453-1474.

[34] G. Weinstein - "On the force between rotating coaxial black holes", Trans. of the Amer. Math. Soc. 343 (1994), p. 899-906.

[35] _ "On the Dirichlet problem for harmonic maps with prescribed singularities.", Duke Math. J. 77 (1995), no. 1, 135-165.

[36] _ " $N$-black hole stationary and axially symmetric solutions of the Einstein/Maxwell equations", Commun. Part. Diff. Eqs. 21 (1996), p. 1389-1430.

[37] - "Harmonic maps with prescribed singularities into Hadamard manifolds", Math. Res. Lett. 3 (1996), p. 835-844.

J.L. CostA, Lisbon University Institute (ISCTE); Mathematical Institute and Magdalen College, Oxford $\bullet$ E-mail : jlca@iscte.pt 